\title{
Stability of Networked Control Systems with Asynchronous Renewal Links: An Impulsive Systems Approach *
}

\author{
Duarte Antunes $^{\text {a,c }}$, João Hespanha ${ }^{\text {b }}$, Carlos Silvestre ${ }^{\text {a }}$ \\ ${ }^{a}$ Dep. of Electrical Eng. and Computer Science, Instituto Superior Técnico, ISR, 1046-001 Lisboa, Portugal \\ ${ }^{\mathrm{b}}$ Dept. of Electrical and Computer Eng., University of California, Santa Barbara, CA 93106-9560, USA. \\ ${ }^{\mathrm{c}}$ Department of Mechanical Engineering, Eindhoven University of Technology, the Netherlands.
}

\begin{abstract}
We consider networked control systems in which sensors, actuators, and controller transmit through asynchronous communication links, each introducing independent and identically distributed intervals between transmissions. We model these scenarios through impulsive systems with several reset maps triggered by independent renewal processes, i.e., the intervals between jumps associated with a given reset map are identically distributed and independent of the other jump intervals. For linear dynamic and reset maps, we establish that mean exponential stability is equivalent to the spectral radius of an integral operator being less than one. We also prove that the origin of a non-linear impulsive system is (locally) stable with probability one if its local linearization about the zero equilibrium is mean exponentially stable, which justifies the importance of studying the linear case. The applicability of the results is illustrated through an example using a linearized model of a batch-reactor.
\end{abstract}

Key words: Networked Control Systems, Stability of Stochastic Systems, Asynchronous Systems, Impulsive Systems.

\section{Introduction}

Consider a networked control system in which a remote controller communicates with a plant through two independent communication links; for example, the actuation data may be sent from the controller to the plant through a shared wired network and the sensor data may be sent from the plant to the controller through a shared

\footnotetext{
* This paper was not presented at any IFAC meeting. Corresponding author D. Antunes, dantunes@isr.ist.utl.pt,Instituto Superior Técnico, ISR, 1046001 Lisboa, Portugal. This work was supported in part by the Fundação para a Ciência e a Tecnologia (Institute for Systems and Robotics/Instituto Superior Técnico plurianual funding) through the PIDDAC Program funds, by Project PTDC/MAR/64546/2006 OBSERVFLY and by the NSF grant \# CNS-0720842. The work of D. Antunes was supported by the Ph.D. Grant SFRH/BD/24632/2005 from Fundação para a Ciência e a Tecnologia and by the Dutch Science Foundation (STW) and the Dutch Organization for Scientific Research (NWO) under the VICI grant "Wireless controls systems: A new frontier in Automation" (No. 11382).
}

Email addresses: dantunes@isr.ist.utl.pt (Duarte Antunes), hespanha@ece.ucsb.edu (João Hespanha), cjs@isr.ist.utl.pt (Carlos Silvestre). wireless network. A reasonable assumption in control over networks utilizing CSMA-type protocols, such as the Ethernet or the Wireless 802.11, is to take the lengths of times needed to gain access to the shared network and to transmit data to be independent and identically distributed (see, e.g. [4], [20], [23]). In the present paper, we show that this networked control system, and, more generally, networked control systems where several sensors, actuators, and controller, are linked through different (wired or/and wireless) networks operating asynchronously, can be modeled by impulsive systems of the following form

$$
\begin{aligned}
& \dot{x}(t)=a(x(t)), \quad t \geq 0, \quad t \neq t_{k}^{\ell}, \quad x(0)=x_{0}, \\
& x\left(t_{k}^{\ell}\right)=j_{\ell}\left(x\left(t_{k}^{\ell-}\right)\right), \quad k \geq 1, \quad \ell \in \mathcal{L}:=\left\{1, \ldots, n_{\ell}\right\},
\end{aligned}
$$

where the duration of the intervals $\left\{h_{k}^{\ell}:=t_{k+1}^{\ell}-t_{k}^{\ell} \mid k \geq\right.$ $0\}$ between jumps times $t_{k}$ associated with a given reset map $j_{\ell}$, are independent and identically distributed and also independent of the jump intervals $\left\{h_{k}^{j} \mid k \geq 0, j \neq\right.$ $\ell, j \in \mathcal{L}\}$. In (1), the notation $x\left(t_{k}^{\ell-}\right)$ indicates the limit from the left of $x$ at the point $t_{k}^{\ell}$ and $n_{\ell}$ denotes the number of reset maps.

We provide stability results for (1), from which one can 
directly infer stability properties for the networked control systems just described. Our main result establishes that when the dynamic map $a$ and the reset maps $j_{\ell}$ are linear, mean exponential stability is equivalent to the spectral radius of an integral operator being less than one, which can be efficiently tested numerically. To prove this result, we first derive conditions for mean exponential stability for (1) with general non-linear dynamic and reset maps. When specialized to linear dynamic and reset maps, these stability conditions can be expressed in terms of the existence of a solution, with certain properties, to an integro-differential equation, which, in turn, is related to the spectral radius of an integral operator. For the general nonlinear case, we show that the origin of (1) is (locally) stable with probability one if the linearization of (1) about zero equilibrium is mean exponentially stable, which justifies the importance of studying the linear case.

To illustrate the applicability of our results, we consider the linearized model of a batch-reactor that appeared in [2], where we assumed that the sensors transmit in a round-robin fashion through a single shared link. We can now test mean exponential stability in the case where the sensors transmit through two asynchronous links both introducing independent and identically distributed intervals between transmissions.

Several references to related work on networked control systems can be found in [13], including systems with asynchronous data transmissions, e.g., [25], and systems with independent and identically distributed intervals between transmission, e.g, [20]. See also [4], [11], [14], [23]. However, the networked control problem we consider here, and the associated class of impulsive systems, seem to have not been studied in the literature. Stability results for deterministic impulsive systems can be found in [12], [19]. The proof of our results builds upon results for piecewise deterministic systems [10] and the stochastic Lyapunov approach [18].

The remainder of the paper is organized as follows. The connection between (1) and networked control systems is given in Section 2. In Section 3 we state and discuss our main results. Our main result concerning the stability of linear impulsive systems, is proved in Section 4; the results concerning mean exponential stability and stability with probability one of (1), are proved in the Appendix. An example is given in Section 5. Final conclusions are given in Section 6. A subset of the results in this paper was presented in the conference paper [3].

Notation: For vectors $a_{i},\left(a_{1}, \ldots, a_{n}\right)$ denotes the column vector $\left[\begin{array}{lll}a_{1}^{\top} & \ldots & a_{n}^{\top}\end{array}\right]^{\top}$. The notation $1_{n}$ indicates a vector of $n$ ones.

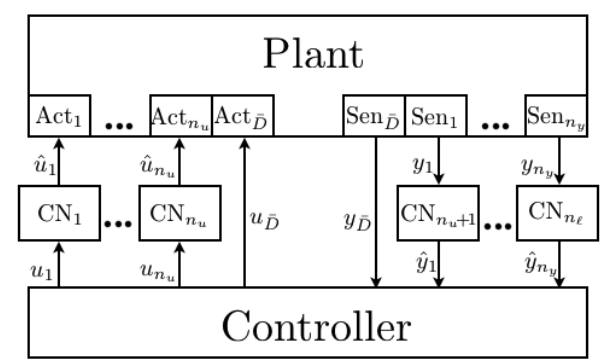

Fig. 1. Networked control setup. Actuators Act ${ }_{j}, 1 \leq j \leq n_{u}$, and sensors $\operatorname{Sen}_{i}, 1 \leq i \leq n_{y}$ are connected to a remote controller though independent communication networks $C N_{\ell}$, $1 \leq \ell \leq n_{\ell}=n_{u}+n_{y}$. Other sensors $\operatorname{Sen}_{\bar{D}}$ and actuators $\operatorname{Act}_{\bar{D}}$ may be directed connected to the controller.

\section{Modeling Networked Control Systems with Impulsive Systems}

Consider a continuous-time plant and a controller described by

$$
\begin{aligned}
& \text { Plant: } \quad \dot{x}_{P}=f_{P}\left(x_{P}, \hat{u}\right), \quad y=g\left(x_{P}\right) \\
& \text { Controller: } \quad \dot{x}_{C}=f_{C}\left(x_{C}, \hat{y}\right), \quad u=h\left(x_{C}, \hat{y}\right) .
\end{aligned}
$$

where the maps $f_{P}, f_{C}, g$ and $h$ are assumed to be differentiable, and $f_{P}$ and $f_{C}$ are assumed to be globally Lipschitz. The controller is assumed to yield the closed-loop stable when the plant and the controller are directly connected, i.e., $\hat{u}(t)=u(t), \hat{y}(t)=y(t)$. However, sensors, actuators, and controller may be spatially distributed and linked via communication networks, in which case this ideal assumption is not valid.

Suppose that there are $\tilde{n}_{y}$ sensors, among which, $n_{y}$ are linked to the controller via $n_{y}$ communication networks, i.e., each sensor transmits through a different network. Then we can partition $y$ as $y=\left(y_{D}, y_{\bar{D}}\right):=$ $\left(g_{D}\left(x_{P}\right), g_{\bar{D}}\left(x_{P}\right)\right)=g\left(x_{P}\right)$ where

$$
y_{D}=\left(y_{1}, \ldots, y_{n_{y}}\right)
$$

comprises the measurement signals $y_{i} \in \mathbb{R}^{s_{i}}, 1 \leq i \leq n_{y}$ of the $n_{y}$ sensors linked to the controller via a network, and $y_{\bar{D}}$ comprises the measurement signals of the sensors whose connection to the controller is ideal. Therefore, partitioning $\hat{y}$ as $\hat{y}=\left(\hat{y}_{1}, \ldots, \hat{y}_{n_{y}}, \hat{y}_{\bar{D}}\right), \hat{y}_{i} \in \mathbb{R}^{s_{i}}$, we have $y_{\bar{D}}(t)=\hat{y}_{\bar{D}}(t)$. Likewise, assuming that there are $\tilde{n}_{u}$ actuators, among which, $n_{u}$ communicate to the plant via a communication network, we can partition $u$ as $u=$ $\left(u_{D}, u_{\bar{D}}\right):=\left(h_{D}\left(x_{C}, \hat{y}\right), h_{\bar{D}}\left(x_{C}, \hat{y}\right)\right)=h\left(x_{C}, \hat{y}\right)$, where

$$
u_{D}=\left(u_{1}, \ldots, u_{n_{u}}\right) \text {, }
$$

comprises the actuation signals $u_{j} \in \mathbb{R}^{r_{j}}, 1 \leq j \leq n_{u}$ of the $n_{u}$ actuators linked to the controller via a network, and $u_{\bar{D}}$ comprises the actuation signals of the actuators whose connection to the controller is ideal. Also here, 
partitioning $\hat{u}$ as $\hat{u}=\left(\hat{u}_{1}, \ldots, \hat{u}_{n_{u}}, \hat{u}_{\bar{D}}\right), \hat{u}_{j} \in \mathbb{R}^{r_{j}}$, we have $u_{\bar{D}}(t)=\hat{u}_{\bar{D}}(t)$. The setup in depicted in Figure 1 .

Let $n_{\ell}:=n_{y}+n_{u}$, and for a given $\ell \in\left\{1, \ldots, n_{y}\right\}$, let $\left\{t_{k}^{\ell} \mid k \geq 0\right\}$ denote the transmission times of the sensor $y_{\ell}$ and for a given $\ell \in\left\{n_{y}+1, \ldots, n_{\ell}\right\}$, let $\left\{t_{k}^{\ell} \mid k \geq 0\right\}$ denote the transmission times of the actuator $u_{\ell-n_{y}}$. Between transmission times we assume that $\hat{y}_{i}$ and $\hat{u}_{j}$ remain constant

$$
\hat{y}_{\ell}(t)=\hat{y}_{\ell}\left(t_{k}^{\ell}\right), \quad t \in\left[t_{k}^{\ell}, t_{k+1}^{\ell}\right), \quad 1 \leq \ell \leq n_{y}
$$

and

$$
\hat{u}_{\ell-n_{y}}(t)=\hat{u}_{\ell-n_{y}}\left(t_{k}^{\ell}\right), \quad t \in\left[t_{k}^{\ell}, t_{k+1}^{\ell}\right), \quad 1 \leq \ell-n_{y} \leq n_{u},
$$

while at transmission times we have the following update equations

$$
\hat{y}_{\ell}\left(t_{k}^{\ell}\right)=y_{\ell}\left(t_{k}^{\ell-}\right), \quad 1 \leq \ell \leq n_{y}
$$

and

$$
\hat{u}_{\ell-n_{y}}\left(t_{k}^{\ell}\right)=u_{\ell-n_{y}}\left(t_{k}^{\ell-}\right), 1 \leq \ell-n_{y} \leq n_{u} .
$$

We assume that in each of the $n_{\ell}$ networks that connect sensors and actuators to the controller, the intervals between transmissions are independent and identically distributed, i.e., $\left\{h_{k}^{\ell}=t_{k+1}^{\ell}-t_{k}^{\ell} \mid k \geq 0\right\}$ are independent and identically distributed random variables, and also independent of the transmission intervals in the remaining networks. Defining,

$$
e:=\left(e_{y}, e_{u}\right):=\left(\hat{y}_{D}-y_{D}, \hat{u}_{D}-u_{D}\right)
$$

and using the fact that we can write

$$
\hat{y}=\left(e_{y}+g_{D}\left(x_{P}\right), g_{\bar{D}}\left(x_{P}\right)\right)
$$

we can model the networked control system (2)-(8) as an impulsive system taking the form (1), where $x=$ $\left(x_{P}, x_{C}, e\right)$ is the state;

$$
a(x)=b(x)\left[\begin{array}{c}
f_{P}\left(x_{P}, \quad\left(e_{u}+h_{D}\left(x_{C}, \hat{y}\right), h_{\bar{D}}\left(x_{C}, \hat{y}\right)\right)\right) \\
f_{C}\left(x_{C}, \quad\left(e_{y}+g_{D}\left(x_{P}\right), g_{\bar{D}}\left(x_{P}\right)\right)\right)
\end{array}\right],
$$

where

$$
b(x)=\left[\begin{array}{cccc}
I & 0 & -\frac{\partial g_{D}}{\partial x_{P}}\left(x_{P}\right)^{\top}-\left(\frac{\partial h_{D}}{\partial y_{\bar{D}}}\left(x_{C}, \hat{y}\right) \frac{\partial g_{\bar{D}}}{\partial x_{P}}\left(x_{P}\right)\right)^{\top} \\
0 & I & 0 & -\frac{\partial h_{D}}{\partial x_{C}}\left(x_{C}, \hat{y}\right)^{\top}
\end{array}\right]^{\top}
$$

and $\hat{y}$ is described by (9), models the plant, controller, and error dynamics; and

$$
j_{\ell}(x)=\left(x_{P}, x_{C}, \hat{j}_{\ell}\left(e_{1}\right), \ldots, \hat{j}_{\ell}\left(e_{n_{\ell}}\right)\right)
$$

models the transmissions at which the error associated with the transmitting sensor/actuator is reset to zero, i.e., $\hat{j}_{\ell}\left(e_{i}\right)=0$, if $i=\ell$, and $\hat{j}_{\ell}\left(e_{i}\right)=e_{i}$, if $i \neq \ell$.

\section{Main results}

We start by providing in Section 3.1 a stability result for (1) with general non-linear dynamic and reset maps. Building upon this result, we are able to establish our main result, presented in Section 3.2, which provides necessary and sufficient stability conditions when the dynamic and reset maps in (1) are linear. In Section 3.3 we relate the stability of the non-linear impulsive system with that of its linearization.

\subsection{Non-linear dynamic and reset maps}

In this section we consider (1) with general maps $a$ and $j_{\ell}$, not necessarily taking the form $(10),(11)$. The maps $a$ and $j_{\ell}, \ell \in \mathcal{L}$ are assumed to be differentiable and globally Lipschitz and the origin is an equilibrium point, i.e., $a(0)=0$ and $j_{\ell}(0)=0, \forall_{\ell \in \mathcal{L}}$. Note that in the special case where $a$ and $j_{\ell}$ are described by (10), (11), this holds if $f_{P}$ and $f_{C}$ are differentiable and globally Lipschitz, the origin is an equilibrium point of both (2) and (3), and $g, h$ are linear maps. We denote by $n$ the dimension of the state $x \in \mathbb{R}^{n}$. We assume that the intervals between jump times $\left\{h_{k}^{\ell}=t_{k+1}^{\ell}-t_{k}^{\ell} \mid k \geq 0\right\}, \ell \in \mathcal{L}$, are described by a probability density function $f_{\ell}(t) \geq 0$, with support in the interval $\left[0, \gamma_{\ell}\right], \gamma_{\ell} \in \mathbb{R}_{>0} \cup\{\infty\}$. Apart from the special case of exponential distributions addressed in Section 3.2, Theorem 7, we assume that the supports are bounded, i.e., $\gamma_{\ell} \neq \infty, \forall_{\ell \in \mathcal{L}}$. This is an important assumption and in Section 6 we give further comments on this. We assume that the $f_{\ell}$ are differentiable ${ }^{1}$ on $\left(0, \gamma_{\ell}\right)$ and we denote the survivor function by

$$
r_{\ell}(s):=\operatorname{Prob}\left[h_{k}^{\ell}>s\right]=\int_{s}^{\gamma_{\ell}} f_{\ell}(r) d r, k \geq 1, s \in\left[0, \gamma_{\ell}\right],
$$

and the hazard rates ${ }^{2}$ by

$$
\lambda_{\ell}\left(\tau_{\ell}\right):=\frac{f_{\ell}\left(\tau_{\ell}\right)}{r_{\ell}\left(\tau_{\ell}\right)}, \quad \tau_{\ell} \in B_{\ell}
$$

where

$$
B_{\ell}:=\left[0, \gamma_{\ell}\right], \quad \ell \in \mathcal{L}
$$

The system (1) is started at $t=0$, with a deterministic initial condition $x_{0}$, where it is subsumed that a time

\footnotetext{
1 We assume differentiability on most functions of interest in the paper to avoid complicating the proofs of our main results.

2 Recall that the hazard rate can be interpreted as $\lambda_{\ell}(\tau)=$ $\lim _{\delta \rightarrow 0} \frac{\left.\text { Prob[Jump in }\left[t_{k}^{\ell}+\tau, t_{k}^{\ell}+\tau+\delta\right) \mid \text { No jump occured in }\left[t_{k}^{\ell}, t_{k}^{\ell}+\tau\right)\right]}{\delta}$
} 
$\tau_{\ell}:=-t_{0}^{\ell}$ has elapsed since the last jump associated with map $\ell$. In other words, we consider that, for each reset map $\ell \in \mathcal{L}$, the first jump times $t_{1}^{\ell}$ satisfy

$$
\operatorname{Prob}\left(\left[t_{1}^{\ell}>s\right]\right)=\frac{r_{\ell}\left(\tau_{\ell}+s\right)}{r_{\ell}\left(\tau_{\ell}\right)}, s \in\left[0, \gamma_{\ell}-\tau_{\ell}\right]
$$

which is the probability that the next jump after $t=0$ occurs after time $s$, given that at $t=0$ a time $\tau_{\ell}$ has elapsed since the map $j$ was triggered.

We need to define the following auxiliary process

$$
v(t)=\left(v_{1}(t), \ldots, v_{n_{\ell}}(t)\right), v_{\ell}(t):=t-t_{k_{\ell}}^{\ell}, v(0)=\tau
$$

where $k_{\ell}:=\max \left\{k \geq 0: t_{k}^{\ell} \leq t\right\}$. The process $v(t)$ keeps track of the time elapsed since the last jump associated with each of the reset maps, and therefore at time $t=0$, $v(0)=\tau=\left(\tau_{1}, \ldots, \tau_{n_{\ell}}\right)=-\left(t_{0}^{1}, \ldots, t_{0}^{n_{\ell}}\right)$. Note that $v(t) \in B$, where

$$
B:=B_{1} \times \cdots \times B_{n_{\ell}} .
$$

and $B_{\ell}$ is described by (14). We also define

$$
\mathbf{x}(t):=(x(t), v(t))
$$

and

$$
\mathbf{x}(0)=\mathbf{x}=:\left(x_{0}, \tau\right) .
$$

As we shall see, $\mathbf{x}$ is a Markov process, although, in general, $x$ is not. In fact, (18) can be constructed as a piecewise deterministic process (cf. Theorem 14 in the Appendix), which allows to establish the following key result in what follows. The proof is given in the Appendix. Let $\pi_{\ell}^{0}$ be a map in $B$ that sets the component $\ell$ of a vector to zero, i.e.,

$$
\pi_{\ell}^{0}: B \mapsto B, \pi_{\ell}^{0}(\tau)=\left(\tau_{1}, \ldots, \tau_{\ell-1}, 0, \tau_{\ell+1}, \ldots, \tau_{n_{\ell}}\right)
$$

Theorem 1 If $V: \mathbb{R}^{n} \times B \mapsto \mathbb{R}$ is a differentiable function, such that

$$
\mathbb{E}\left[\sum_{t_{k}^{\ell} \leq n}\left|V\left(\mathbf{x}\left(t_{k}^{\ell}\right)\right)-V\left(\mathbf{x}\left(t_{k}^{\ell-}\right)\right)\right|\right]<\infty, \quad \forall_{n \in \mathbb{N}}
$$

where $\ell \in \mathcal{L}$, then for the system (1), (16), (18) with initial condition (19), we have that

$$
\mathbb{E}[V(\mathbf{x}(t))]=V(\mathbf{x})+\mathbb{E} \int_{0}^{t} \mathfrak{A} V(\mathbf{x}(s)) d s, \quad \forall_{t \geq 0},
$$

where

$$
\begin{aligned}
& \mathfrak{A} V(\mathbf{x}):=\frac{\partial}{\partial \tau} V(\mathbf{x})+ \\
& \mathfrak{X}_{x} V(\mathbf{x})+\sum_{\ell=1}^{n_{\ell}} \lambda_{\ell}\left(\tau_{\ell}\right)\left[V\left(\left(j_{\ell}(x), \pi_{\ell}^{0}(\tau)\right)\right)-V(\mathbf{x})\right],
\end{aligned}
$$

for $\mathbf{x}=(x, \tau) \in \mathbb{R}^{n} \times B$, and $\mathfrak{X}_{x} V(\mathbf{x}):=\sum_{i=1}^{n} \frac{\partial V(\mathbf{x})}{\partial x_{i}} a_{i}(x)$.

We consider the following definition of stability for (1).

Definition 2 We say that (1) is mean exponentially stable (MES) if there exists constants $c>0, \alpha>0$ such that for every initial condition $x_{0}$, the following holds

$$
\mathbb{E}\left[x(t)^{\top} x(t)\right] \leq c e^{-\alpha t} x_{0}^{\top} x_{0}, \forall_{t \geq 0} .
$$

The following result establishes general conditions for (1) to be MES, providing a stochastic analog of a well known result for deterministic non-linear systems (cf. [16, Th.4.10]). The proof can be found in the Appendix.

Theorem 3 The system (1) is MES if and only if there exists a differentiable positive function $V: \mathbb{R}^{n} \times B \mapsto$ $\mathbb{R}_{\geq 0}$ which equals zero at zero, and positive constants $c_{1}, c_{2}, r$ such that for every $\mathbf{x}=(x, \tau) \in \mathbb{R}^{n} \times B$,

$$
\begin{gathered}
c_{1}\|x\|^{2} \leq V(\mathbf{x}) \leq c_{2}\|x\|^{2}, \\
\mathfrak{A} V(\mathbf{x}) \leq-r\|x\|^{2} .
\end{gathered}
$$

\subsection{Linear dynamic and reset maps}

In this subsection, we consider the following linear version of (1)

$$
\begin{aligned}
& \dot{x}(t)=A x(t), \quad t \geq 0, \quad t \neq t_{k}^{\ell}, \quad x(0)=x_{0}, \\
& x\left(t_{k}^{\ell}\right)=J_{\ell} x\left(t_{k}^{\ell-}\right), \quad k \geq 1, \quad \ell \in \mathcal{L},
\end{aligned}
$$

where $\left\{h_{k}^{\ell}=t_{k+1}^{\ell}-t_{k}^{\ell} \mid k \geq 0\right\}$ and $t_{0}^{\ell}$ are as described for (1) and for which we consider (16) and (18) with initial condition (19).

Define a variable $\tau=\left(\tau_{1}, \ldots, \tau_{n_{\ell}}\right) \in B$ where $\tau_{j} \in B_{j}$. Furthermore, let $\hat{B}_{j}=B_{1} \times \ldots B_{j-1} \times B_{j+1} \times \cdots \times B_{n_{\ell}}$ and define the map $\pi_{\ell}$ that removes the component $\ell$ from the vector $\tau$, i.e., $\pi_{\ell}: B \mapsto \hat{B}_{\ell}, \pi_{\ell}(\tau)=$ $\left(\tau_{1}, \ldots, \tau_{\ell-1}, \tau_{\ell+1}, \ldots, \tau_{n_{\ell}}\right)$. Let $\mathcal{P}_{a}$ be the Cartesian product space of $n_{\ell}$ measurable real symmetric matrix functions, each defined in $\hat{B}_{\ell}$, i.e., if 
$P=\left(P_{1}\left(\hat{\tau}_{1}\right), \ldots, P_{n_{\ell}}\left(\hat{\tau}_{n_{\ell}}\right)\right) \in \mathcal{P}_{a}$, then $P_{\ell}\left(\hat{\tau}_{\ell}\right)^{\top}=$ $P_{\ell}\left(\hat{\tau}_{\ell}\right), \forall \hat{\tau}_{l} \in \hat{B}_{\ell}$. Sum and multiplication by scalar in $\mathcal{P}_{a}$ are defined in a natural way and we consider the usual inner product $\langle Q, R\rangle=\sum_{\ell=1}^{n_{\ell}} \int_{\hat{B}_{\ell}} Q_{\ell}\left(\hat{\tau}_{\ell}\right)^{\top} R_{\ell}\left(\hat{\tau}_{\ell}\right) d \hat{\tau}_{\ell}$ for $Q, R \in \mathcal{P}$. Then we define the space $\mathcal{P}$ as the space of elements $P \subset \mathcal{P}_{a}$ such that $\langle P, P\rangle\langle\infty$, which can be shown to be a Hilbert space. Let $\mathfrak{L}: \mathcal{P} \mapsto \mathcal{P}$ be the following integral operator

$$
\left(Q_{1}, \ldots, Q_{n_{\ell}}\right)=\mathfrak{L}\left(P_{1}, \ldots, P_{n_{\ell}}\right)
$$

where $Q_{\ell}\left(\pi_{\ell}(\tau)\right):=R\left(\pi_{\ell}^{0}(\tau)\right)$,

$$
\begin{aligned}
& R(\tau):= \\
& \sum_{\ell=1}^{n_{\ell}} \int_{0}^{\bar{\gamma}}\left(J_{\ell} e^{A s}\right)^{\top} P_{\ell}\left(\pi_{\ell}(\tau)+s 1_{n_{\ell}-1}\right) J_{\ell} e^{A s} \frac{\hat{r}_{\ell}(\tau, s)}{\bar{r}_{\ell}(\tau)} \frac{f_{\ell}\left(\tau_{\ell}+s\right)}{r_{\ell}\left(\tau_{\ell}\right)} d s,
\end{aligned}
$$

$\hat{r}_{\ell}(\tau, s):=\Pi_{j=1, j \neq \ell}^{n_{\ell}} r_{j}\left(\tau_{j}+s\right), \bar{r}_{\ell}(\tau):=\prod_{j=1, j \neq \ell}^{n_{\ell}} r_{j}\left(\tau_{j}\right)$, $\bar{\gamma}:=\min \left\{\gamma_{\ell}-\tau_{\ell} \mid \ell \in \mathcal{L}\right\}$ and $1_{n_{\ell}-1}$ is a vector with $n_{\ell}-1$ components set to one.

For example for $n_{\ell}=1,(28),(29)$ take the form

$$
Q_{1}=\int_{0}^{\gamma_{1}}\left(J_{1} e^{A s}\right)^{\top} P_{1} J_{1} e^{A s} f_{1}(s) d s,
$$

where $P_{1}$ and $Q_{1}$ are symmetric matrices, and in this special case $\mathcal{P}$ is a finite dimensional space since its elements are matrices and not matrix-valued functions. For $n_{\ell}=2$, we have

$$
\begin{aligned}
& Q_{1}\left(\tau_{2}\right)=\int_{0}^{\bar{\gamma}_{1}}\left(J_{1} e^{A s}\right)^{\top} P_{1}\left(\tau_{2}+s\right) J_{1} e^{A s} \frac{r_{2}\left(\tau_{2}+s\right)}{r_{2}\left(\tau_{2}\right)} f_{1}(s) d s \\
&+\int_{0}^{\bar{\gamma}_{1}}\left(J_{2} e^{A s}\right)^{\top} P_{2}(s) J_{2} e^{A s} r_{1}(s) \frac{f_{2}\left(\tau_{2}+s\right)}{r_{2}\left(\tau_{2}\right)} d s, \\
& \tau_{2} \in\left[0, \gamma_{2}\right], \\
& Q_{2}\left(\tau_{1}\right)= \int_{0}^{\bar{\gamma}_{2}}\left(J_{2} e^{A s}\right)^{\top} P_{2}\left(\tau_{1}+s\right) J_{2} e^{A s} \frac{r_{1}\left(\tau_{1}+s\right)}{r_{1}\left(\tau_{1}\right)} f_{2}(s) d s \\
&+\int_{0}^{\bar{\gamma}_{2}}\left(J_{1} e^{A s}\right)^{\top} P_{1}(s) J_{1} e^{A s} r_{2}(s) \frac{f_{1}\left(\tau_{1}+s\right)}{r_{1}\left(\tau_{1}\right)} d s . \\
& \tau_{1} \in\left[0, \gamma_{1}\right],
\end{aligned}
$$

where $\bar{\gamma}_{1}=\min \left(\gamma_{1}, \gamma_{2}-\tau_{2}\right)$, and $\bar{\gamma}_{2}=\min \left(\gamma_{1}-\tau_{1}, \gamma_{2}\right)$.

Since $\mathfrak{L}$ operates in a real space $\mathcal{P}$, to define its spectral radius we consider the complexification of $\mathcal{P}$ (cf. [17, p. 77]), i.e., the space $\tilde{\mathcal{P}}:=\{Q=P+i R: P, R \in \mathcal{P}\}$. For $Q=P+i R \in \tilde{\mathcal{P}}$, one defines $\mathfrak{L}(Q):=\mathfrak{L}(P)+i \mathfrak{L}(R)$. The spectral radius is defined as follows:

$$
r_{\sigma}(\mathfrak{L}):=\max \{|\lambda|: \lambda \in \sigma(\mathfrak{L})\}
$$

where $\sigma(\mathfrak{L}):=\{\lambda \in \mathcal{C}: \mathfrak{L}-\lambda$ I is not invertible in $\tilde{\mathcal{P}}\}$ denotes the spectrum and I the identity. Note that, defining $\mathcal{P}$ as a real space, and defining the spectral radius of $\mathcal{L}$ acting on $\mathcal{P}$ as in (32) is generally different from considering $\mathcal{P}$ to be a complex space, where the matrix components $P_{\ell}$ of $P=\left(P_{1}, \ldots, P_{n_{\ell}}\right) \in \mathcal{P}$ are self-adjoint matrices, and defining the spectral radius of $\mathcal{L}$ as usual. We shall use the first construction since this will allow us to readily use the results for positive operators given in [17] to prove our results in Section 4.

The following is the main result of the paper.

Theorem 4 The system (27) is MES if and only if $r_{\sigma}(\mathfrak{L})<1$.

The theorem is proved in Section 4 . We discuss next how one can numerically compute $r_{\sigma}(\mathfrak{L})$, and some special cases of the impulsive system, for which one can provide alternative stability conditions to Theorem 4 .

\section{Computation of the spectral radius of $\mathfrak{L}$}

One can show that $\mathfrak{L}$ is a compact operator (using, e.g., [8, p. 165, Th. 4.1]) and therefore its spectrum consists either of a finite set of eigenvalues $\lambda: \mathfrak{L} P=\lambda P$ for some $P \in \tilde{\mathcal{P}}$ or of a countable set of eigenvalues with no accumulation point other than zero (cf., e.g., [8, p. 117, Th. 2.34]). For simplicity, consider first the case $n_{\ell}=2$, in which $\mathfrak{L}$ is described by (31). A numerical method to compute $r_{\sigma}(\mathfrak{L})$ is the following. Take a grid of points $\bar{\tau}_{1 j} \in\left[0, \gamma_{1}\right], 1 \leq j \leq n_{d 1}$, and $\bar{\tau}_{2 j} \in$ $\left[0, \gamma_{2}\right], 1 \leq j \leq n_{d 2}$, and consider the map obtained by replacing $P_{1}\left(\tau_{2}\right), P_{2}\left(\tau_{1}\right)$ in $(31)$ by interpolating piecewise polynomials at the points $\left\{P_{1}\left(\bar{\tau}_{2 k}\right), P_{2}\left(\bar{\tau}_{1 k}\right)\right\}$, and evaluating the integrals (31) at the grid points to obtain $\left\{Q_{1}\left(\bar{\tau}_{2 k}\right), Q_{2}\left(\bar{\tau}_{1 k}\right)\right\}$. This yields a finite rank operator, from the chosen space of piecewise polynomials in $\mathcal{P}$ to itself, and assuming $n_{d_{1}}=n_{d_{2}}=n_{d}$, its matrix representation has dimension $2 n_{d}^{2} n(n+1) / 2$, since $P_{i}, Q_{i}, i \in\{1,2\}$ are symmetric. Denote by $L_{n_{d}}$ the finite rank operator obtained and by $\mathcal{P}_{n_{d}}$ the space of piecewise polynomials described above. The method just described is known as the collocation method, a special case of the projection method (cf. [8, p. 177]), and one can conclude from the results in [8] that $r_{\sigma}\left(L_{n_{d}}\right) \rightarrow r_{\sigma}(\mathfrak{L})$ as $n_{d} \rightarrow \infty$, for typical piecewise polynomial approximations, such as the trapezoidal or the zero order approximation. ${ }^{3}$ For general $n_{\ell}$, the space of piecewise

\footnotetext{
3 In fact, from the spectral characterization of compact operators described above, one can conclude from [8, p.232, Th. 5.5 and p.250, Example 5.14]), that the eigenvalues of the compact operators $L_{n_{d}}$ converge to the eigenvalues of the compact operator $\mathfrak{L}$ which allows us to conclude that $r_{\sigma}\left(L_{n_{d}}\right) \rightarrow r_{\sigma}(\mathfrak{L})$.
} 
polynomials $\mathcal{P}_{n_{d}}$ can be defined in a similar way and this method involves computing the spectral radius of a $n_{d}^{n_{\ell}} n_{\ell} n(n+1) / 2$ matrix, which means that computing $r_{\sigma}(\mathfrak{L})$ may require significant computational effort when the number of reset maps is large.

From the results in $[8$, Sec. $6.1,6.2]$, one can conclude that, for the projection method just described, the distance between an eigenvalue $\lambda_{n_{d}}$ of $L_{n_{d}}$ and the corresponding eigenvalue $\lambda$ of $\mathfrak{L}$ that $\lambda_{n_{d}}$ approximates is dictated by

$$
\left.\alpha_{n_{d}}:=\sup \left\{\left\|\left(I-\Pi_{n_{d}}\right) \psi\right\|\right) \mid \psi \in \mathcal{M}_{\lambda},\|\psi\|=1\right\},
$$

where $\Pi_{n_{d}}$ is the projection from $\tilde{\mathcal{P}}$ to $\mathcal{P}_{n_{d}}$, and $\mathcal{M}_{\lambda} \subseteq \tilde{\mathcal{P}}$ is the invariant subspace associated with the eigenvalue $\lambda$, which in the special case in which the algebraic and geometric multiplicities of $\lambda$ coincide (see $[8$, p. 97,108] for the definitions) is described by

$$
\mathcal{M}_{\lambda}:=\{\Psi \in \tilde{\mathcal{P}}: \mathfrak{L} \Psi=\lambda \Psi\} .
$$

In fact, the convergence of $\lambda_{n_{d}}$ to $\lambda$ as $n_{d} \rightarrow \infty$ and $\alpha_{n_{d}} \rightarrow 0$ is at least of order $\alpha_{n_{d}}$ (cf. [8, Lem. 6.9, 6.10]), i.e.,

$$
\left|\lambda-\lambda_{n_{d}}\right| \leq c_{1} \alpha_{n_{d}}^{p}, \quad p=1
$$

for some constant $c_{1}>0$, and often quadratic with $\alpha_{n_{d}}$ $(p=2$ in (34)) under mild assumptions on the projection $\Pi_{n_{d}}$ (cf. [8, Th. 6.11]). Due to the spectral characterization of compact operators this implies the same convergence properties for $\left|r_{\sigma}\left(L_{n_{d}}\right)-r_{\sigma}(\mathfrak{L})\right|$. The impact that the number of grid points $n_{d}$ has on the approximation of $r_{\sigma}(\mathfrak{L})$ is therefore encapsulated on the dependency of $\alpha_{n_{d}}$ on $n_{d}$. As an example, suppose that $n_{\ell}=2, \mathcal{P}_{n_{d}}$ corresponds to the set of zero-order interpolating piecewise polynomials, and that there exists only one eigenvalue with the same norm as the spectral radius $\lambda:|\lambda|=r_{\sigma}(\mathfrak{L})$ and with the same geometric and algebraic multiplicities. Then it is possible to conclude that a function $\Psi \in \mathcal{M}_{\lambda}$, i.e., a function of the form $\Psi(\tau)=\left(\Psi_{1}\left(\tau_{2}\right), \Psi_{2}\left(\tau_{1}\right)\right) \in \tilde{\mathcal{P}}$ such that $\mathfrak{L} \Psi=\lambda \Psi$, where $\mathfrak{L}$ is described by (31), must be differentiable. Since, for a differentiable function $\Psi$, the norm of the error of a piecewise zero order approximation $\left\|\left(I-\Pi_{n_{d}}\right)(\Psi)\right\|$ is bounded by a linear function of $\frac{1}{n_{d}}$ (cf., e.g., [8, p. 167]) one can conclude that

$$
\alpha_{n_{d}} \leq \frac{c_{2}}{n_{d}}
$$

for some $c_{2}>0$. We refer to [8] for more elaborate techniques to compute the spectral radius of an integral operator, including the iterative refinement method, and further error bound results.

\section{Special cases}

A first special case is when there is only one reset map, i.e., $n_{\ell}=1$. In this case $\mathcal{P}$ is simply the finite dimensional space of symmetric matrices and $\mathcal{L}$ is the linear map $P_{1} \mapsto Q_{1}$ between two finite dimensional space defined in $(30)$. In this case $r_{\sigma}(\mathfrak{L})<1$ reduces to testing if the spectral radius of the following matrix is less than one

$$
M_{1}:=\int_{0}^{\gamma_{1}}\left(J_{1} e^{A s}\right)^{\top} \otimes\left(J_{1} e^{A s}\right)^{\top} f_{1}(s) d s
$$

This condition is also obtained in [4], where the case $n_{\ell}=1$ is analyzed using a different approach, based on Volterra equations, which does not appear to generalize to the problem considered in this paper.

A second special case is when the maps $A, J_{\ell}$ commute, i.e., $A J_{\ell}=J_{\ell} A$, and $J_{\ell} J_{r}=J_{r} J_{\ell}, \forall_{\ell, r \in \mathcal{L}}$. Although this is generally not the case for the linear matrices obtained from (10) and (11) when the dynamics of (2) and (3) are linear, this special case is still of interest for the general model (27). In this case, the following result, proved in the Appendix, provides alternative stability conditions to Theorem 4.

Theorem 5 When the maps $A$ and $J_{\ell}, \ell \in \mathcal{L}$ commute, the system (27) is MES if

$$
2 \bar{\lambda}+\sum_{\ell=1}^{n_{\ell}} \alpha_{\ell}<0
$$

where $\bar{\lambda}$ is the maximum real part of the eigenvalues of $A$ and the $\alpha_{\ell}$ are given by

$\alpha_{\ell}=\left\{\begin{array}{l}-\infty, \text { if } r_{\sigma}\left(J_{\ell}^{\top} \otimes J_{\ell}^{\top}\right)=0 \\ a \in \mathbb{R}: \int_{0}^{\gamma_{\ell}} e^{-a s} f_{\ell}(s) d s=\frac{1}{r_{\sigma}\left(J_{\ell}^{\top} \otimes J_{\ell}^{\top}\right)}, \text { otherwise }\end{array}\right.$

where $\otimes$ denotes the Kronecker product.

It is important to emphasize, that even for the commuting case, the condition (35) is sufficient but not necessary, as shown in the following example.

Example 6 Suppose that $A=[0]_{2 \times 2}, n_{\ell}=1, J_{1}=$ $\left[\begin{array}{cc}a_{1} & 0 \\ 0 & 0\end{array}\right]$, and $J_{2}=\left[\begin{array}{cc}0 & 0 \\ 0 & a_{2}\end{array}\right]$, where $a_{1}>1, a_{2}>1$. Then, $\bar{\lambda}=0$ and, from (36), we can conclude that $\alpha_{1}>0$ and $\alpha_{2}>0$. Thus, (35) does not hold. However, since the state remains constant between jump times $t_{k}^{\ell}, \ell \in\{1,2\}$, and component $i$ is reset to zero when the reset map $i$ is triggered, $i \in\{1,2\}$, we conclude that $\mathbb{E}\left[x(t)^{\top} x(t)\right]=$ $0, t>\max \left(\gamma_{1}, \gamma_{2}\right)$ and therefore $(27)$ is $M E S$. 
A third special case is when the probability densities $f_{\ell}$ correspond to exponential distributions, i.e., $r_{\ell}(y)=$ $e^{-\beta_{\ell} y}$. Since the support of the probability density functions is not bounded we assume the following

$$
2 \bar{\lambda}<\sum_{\ell=1}^{n_{\ell}} \beta_{\ell},
$$

where $\bar{\lambda}$ is the maximum real part of the eigenvalues of $A$. This assumption can be shown to assure that the expected value of a quadratic function of the state of the system does not go unbounded between jump times. This assertion can be obtained using a similar reasoning to $[4, T h .3]$. The next theorem states that, in this case, we can provide stability conditions in the form of LMIs. The proof is omitted due to space limitations but can be found in $[1$, Ch.4].

Theorem 7 Suppose that $r_{\ell}(y)=e^{-\beta_{\ell} y}$ and that (37) holds. Then the system (27) is MES if and only if

$$
\exists_{P>0}: A^{\boldsymbol{\top}} P+P A+\sum_{\ell=1}^{n_{\ell}} \beta_{\ell}\left(J_{\ell}^{\top} P J_{\ell}-P\right)<0 .
$$

\subsection{Stability with probability one}

The following definition is adapted from [18].

Definition 8 We say that the origin of the system (1) is (locally) stable with probability one if for every $\rho>0$ and $\epsilon>0$ there is a $\delta(\rho, \epsilon)>0$ such that, if $\left\|x_{0}\right\|<\delta(\rho, \epsilon)$ then

$$
\operatorname{Prob}\left\{\sup _{\infty>t \geq 0}\|x(t)\| \geq \epsilon\right\} \leq \rho .
$$

The following result shows that one can assert stability with probability one of the origin of (1), by establishing mean exponential stability for its linearization, which can be tested by Theorem 4 . The proof is provided in the Appendix.

Theorem 9 If (27) is MES with $A=\frac{\partial}{\partial x} a(x)_{\left.\right|_{x=0}}$ and $J_{\ell}=\frac{\partial}{\partial x} j_{\ell}(x)_{\mid x=0}, \ell \in \mathcal{L}$ where $a$ and $j_{\ell}$ are the nonlinear maps in (1), then the origin of (1) is stable with probability one.

This theorem allows us to conclude a property analogous to the one proved in [19] stating that a standard periodic sampled-data connection of a non-linear plant and a non-linear controller is locally stable if the sampleddata connection of the linearization of the plant and of the linearization of the controller is stable. In fact, from Theorem 9, we can conclude that in the setup of Section 2, the non-linear networked control system described by $(10),(11)$ is stable with probability one, if the networked control system obtained by replacing $f_{P}, g$, $f_{C}$, and $h$, by their local linearizations about the zero equilibrium is mean exponentially stable, which can be tested by Theorem 4 .

\section{Proof of Theorem 4}

We prove Theorem 4 through three steps: (i) we show that specializing the stability conditions of Theorem 3 to the system (27), yields mean square stability conditions for (27) in terms of the existence of a solution, with certain properties, to an integro-differential equation; (ii) we establish that these conditions are equivalent to the existence of a solution, with certain properties, to a Fredholm equation; (iii) we prove that (ii) is equivalent to the spectral radius of the integral operator of the Fredholm equation being less than one.

\section{(i) MES for Linear impulsive systems}

The Theorem 3 can be specialized to (27) as follows.

Theorem 10 The system (27) is MES if and only if for every differentiable symmetric matrix functions $Y(\tau)$ and $Z(\tau)$ such that $a_{1} \leq Y(\tau) \leq a_{2}, \forall_{\tau \in B}$, and $b_{1} \leq$ $Z(\tau) \leq b_{2}, \forall_{\tau \in B}$, there exists a differentiable symmetric matrix function $X(\tau)$, such that $c_{1} \leq X(\tau) \leq c_{2}, \forall_{\tau \in B}$, and for every $\tau \in B$,

$$
\begin{aligned}
& \sum_{\ell=1}^{n_{\ell}} \frac{\partial}{\partial \tau_{\ell}} X(\tau)+A^{\top} X(\tau)+X(\tau) A+ \\
& \sum_{\ell=1}^{n_{\ell}} \lambda_{\ell}\left(\tau_{\ell}\right)\left[J_{\ell}^{\top} X\left(\pi_{\ell}^{0}(\tau)\right) J_{\ell}-X(\tau)+Z(\tau)\right]+Y(\tau)=0,
\end{aligned}
$$

where $\pi_{\ell}^{0}$ is defined by $(20)$, and $a_{i}, b_{i}, c_{i}, i \in\{1,2\}$ are positive constants.

Proof To prove sufficiency we use Theorem 3 and consider the function $V(\mathbf{x}(t))=x^{\top}(t) X(v(t)) x(t)$ where $\mathbf{x}=(x, v)$ and $X(\tau), \tau \in B$ satisfies (40) and the remaining conditions of the theorem. Then from (23) we have that

$$
\begin{aligned}
\mathfrak{A}\left(x^{\top} X(v) x\right) & =x^{\top}\left[\sum_{\ell=1}^{n_{\ell}} \frac{\partial}{\partial \tau_{\ell}} X(v)+A^{\top} X(v)+X(v) A+\right. \\
& \left.+\sum_{\ell=1}^{n_{\ell}} \lambda_{\ell}\left(v_{\ell}\right)\left[J_{\ell}^{\top} X\left(\pi_{\ell}^{0}(v)\right) J_{\ell}-X(v)\right]\right] x
\end{aligned}
$$


Using (40) we obtain

$\mathfrak{A}\left(x^{\top} X(v) x\right)=-x^{\top}\left[\left(\sum_{\ell=1}^{n_{\ell}} \lambda_{\ell}\left(v_{\ell}\right) Z(v)+Y(v)\right)\right] x \leq-a_{1}\|x\|^{2}$,

and since $c_{1} I \leq X(v) \leq c_{2} I$, we have that $c_{1}\|x\|^{2} \leq$ $V(\mathbf{x}) \leq c_{2}\|x\|^{2}$. Using Theorem 3, applied to (27), we conclude that (27) is MES.

Necessity follows by using the same arguments as in the proof of Theorem 3 and noticing that the function (75) takes the form $V(\mathrm{x})=x_{0}^{\top} X(\tau) x_{0}$, for $\mathrm{x}=\left(x_{0}, \tau\right) \in$ $\mathbb{R}^{n} \times B$

$$
X(\tau)=X_{1}(\tau)+X_{2}(\tau)
$$

where

$$
\begin{aligned}
& X_{1}(\tau)=\int_{0}^{+\infty} \mathbb{E}_{\tau}\left[\Phi(t)^{\top} Y(v(t)) \Phi(t)\right] d t, \\
& X_{2}(\tau)=\sum_{k>0, \ell \in \mathcal{L}} \mathbb{E}_{\tau}\left[\Phi\left(t_{k}^{\ell}\right)^{\top} Z\left(v\left(t_{k}^{\ell}\right)\right) \Phi\left(t_{k}^{\ell}\right)\right],
\end{aligned}
$$

$\Phi(t)$ is the transition matrix of the system (27), i.e.,

$$
\Phi(t)=e^{A\left(t-t_{r}^{\ell_{r}}\right)} J_{\ell_{r-1}} \ldots J_{\ell_{1}} e^{A h_{1}^{\ell_{1}}} J_{\ell_{0}} e^{A h_{0}^{\ell_{0}}},
$$

where $\left\{\ell_{j} \in \mathcal{L}, j \geq 0\right\}$ is the triggered sequence of reset maps, $r=\max \left\{k: t_{k} \leq t\right\}$ and $\mathbb{E}_{\tau}$ emphasizes that expectation subsumes that the process $\Phi(t)$ depends on the initial conditions $\tau$ of the process $v(t)$. Since from Theorem 3, $c_{1}\left\|x_{0}\right\|^{2} \leq V(\mathrm{x}) \leq c_{2}\left\|x_{0}\right\|^{2}$ it follows that $c_{1} I \leq X(\tau) \leq c_{2} I$. From [10, p.92, Th.(32.2)]) it follows that

$$
\mathfrak{A}\left(x_{0}^{\top} X_{1}(\tau) x_{0}\right)=-x_{0}^{\top} Y(\tau) x_{0}
$$

and from $[10$, p.90,91] we have that

$$
x_{0}^{\top} X_{2}(\tau) x_{0}=\mathbb{E}\left[\int_{0}^{\infty} \lambda_{T}(v(t)) x(t)^{\top} Z(v(t)) x(t)\right],
$$

where

$$
\lambda_{T}(\tau):=\sum_{j=1}^{n_{\ell}} \lambda_{j}\left(\tau_{j}\right),
$$

from which one can conclude again from $[10$, p.92, Th. (32.2)] that

$$
\mathfrak{A}\left(x_{0}^{\top} X_{2}(\tau) x_{0}\right)=-\lambda_{T}(\tau) x_{0}^{\top} Z(\tau) x_{0} .
$$

Thus, for every $\left(x_{0}, \tau\right)$, we have

$$
\mathfrak{A}\left(x_{0}^{\top} X(\tau) x_{0}\right)=-x_{0}^{\top}\left(Y(\tau)+\lambda_{T}(\tau) Z(\tau)\right) x_{0}
$$

Computing $\mathfrak{A}\left(x^{\top} X(v) x\right)$ from (23) we obtain (41) which must be equal to $(46)$ when $\mathrm{x}=\left(x_{0}, \tau\right)$ is replaced by $\mathbf{x}=(x, v)$, from which we conclude $(40)$. (ii) Fredholm equation

Let $\mathcal{U}$ be the space of elements $\left(U_{1}\left(\hat{\tau}_{1}\right), \ldots, U_{n_{\ell}}\left(\hat{\tau}_{n_{\ell}}\right)\right) \in$ $\mathcal{P}$ for which $U_{\ell}\left(\hat{\tau}_{\ell}\right) \geq 0, \forall_{\ell \in \mathfrak{L}}, \forall_{\hat{\tau}_{\ell} \in B_{\ell}}$. The space $\mathcal{V} \subset \mathcal{U}$ is defined similarly but requiring $U_{\ell}\left(\hat{\tau}_{\ell}\right)>0, \forall \forall_{\ell \in \mathfrak{L}}, \forall_{\hat{\tau}_{\ell} \in B_{\ell}}$.

Theorem 11 The system (27) is MES if and only if for every differentiable symmetric matrix functions $Y(\tau)$ and $Z(\tau)$ such that $a_{1} \leq Y(\tau) \leq a_{2}, \forall_{\tau \in B}$, and $b_{1} \leq$ $Z(\tau) \leq b_{2}, \forall_{\tau \in B}$, there exists a solution $P \in \mathcal{V}$ to the Fredholm equation

$$
P=\mathfrak{L}(P)+U
$$

where $U=\left(U_{1}, \ldots, U_{n_{\ell}}\right)$,

$$
U_{\ell}\left(\pi_{\ell}(\tau)\right):=W\left(\pi_{\ell}^{0}(\tau)\right)
$$

and

$$
\begin{aligned}
W(\tau):= & \sum_{\ell=1}^{n_{\ell}} \int_{0}^{\bar{\gamma}} e^{A^{\top} s} Z\left(\tau+s 1_{n_{\ell}}\right) e^{A s} \frac{\hat{r}_{\ell}(\tau, s)}{\bar{r}_{\ell}(\tau)} \frac{f_{\ell}\left(\tau_{\ell}+s\right)}{r_{\ell}\left(\tau_{\ell}\right)} d s+ \\
& \int_{0}^{\bar{\gamma}} e^{A^{\top} s} Y\left(\tau+s 1_{n_{\ell}}\right) e^{A s} \Pi_{\ell=1}^{n_{\ell}} \frac{r_{\ell}\left(\tau_{\ell}+s\right)}{r_{\ell}\left(\tau_{\ell}\right)} d s
\end{aligned},
$$

and $a_{i}, b_{i}, i \in\{1,2\}$ are positive constants.

Proof Suppose that (27) is MES and therefore there exists a solution $X$ to (40) given by (42). Let

$$
P\left(\pi_{\ell}(\tau)\right):=X\left(\pi_{\ell}^{0}(\tau)\right), \quad \pi_{\ell}(\tau) \in B_{\ell}
$$

We prove next that $X(\tau)$ satisfies

$$
\begin{aligned}
& X(\tau)=W(\tau)+ \\
& \sum_{\ell=1}^{n_{\ell}} \int_{0}^{\gamma_{\ell}}\left(J_{\ell} e^{A s}\right)^{\top} X\left(\pi_{\ell}^{0}\left(\tau+s 1_{n_{\ell}}\right)\right) J_{\ell} e^{A s} \frac{\hat{r}_{\ell}(\tau, s)}{\bar{r}_{\ell}(\tau)} \frac{f_{\ell}\left(\tau_{\ell}+s\right)}{r_{\ell}\left(\tau_{\ell}\right)} d s .
\end{aligned}
$$

Then sufficiency follows by directly using (50) in (49), and noticing that the fact that $X(\tau)$, given by (42), satisfies $X(\tau) \geq c_{1} I, \forall_{\tau \in B}, c_{1}>0$, implies that $P \in \mathcal{V}$.

To this effect, we start by conditioning the integrand in (43) on the time of the first jump $t_{1}:=\min \left\{t_{1}^{\ell}, \ell \in \mathcal{L}\right\}$,

$$
\begin{array}{r}
\mathbb{E}_{\tau}\left[\Phi(t)^{\top} Y(v(t)) \Phi(t)\right]=\mathbb{E}_{\tau}\left[\left(\Phi(t)^{\top} Y(v(t)) \Phi(t)\right) \mathbf{1}_{\left[t_{1}>t\right]}\right] \\
+\sum_{\ell=1}^{n_{\ell}} \mathbb{E}_{\tau}\left[\left(\Phi(t)^{\top} Y(v(t)) \Phi(t)\right) \mathbf{1}_{C_{\ell}(t)}\right]
\end{array}
$$

where $C_{\ell}(t)=\left[\min \left\{t_{1}^{j}, j \in \mathcal{L}\right\}=t_{1}^{\ell}=t_{1} \leq t\right] \wedge\left[t_{1}^{j}>\right.$ $\left.t_{1}, j \neq \ell\right]$, is the event that jump $\ell$ is the first to trigger, given that a trigger occurred before time $t$. Using (15) 
the first term on the right hand side of (51) is given by $e^{A^{\top} t} Y\left(\tau+t 1_{n_{\ell}}\right) e^{A t} \Pi_{\ell=1}^{n_{\ell}} \frac{r_{\ell}\left(\tau_{\ell}+t\right)}{r_{\ell}\left(\tau_{\ell}\right)}$. Note that for a function $G\left(t_{1}\right)$,

$$
\begin{aligned}
\mathbb{E}_{\tau}\left[G\left(t_{1}\right) \mathbf{1}_{C_{\ell}(t)}\right] & =\int_{0}^{t} \mathbb{E}\left[G(s) \mathbf{1}_{\left[t_{1}^{j}>s, j \neq \ell\right]} \mid t_{1}^{\ell}=s\right] \frac{f_{\ell}\left(\tau_{\ell}+s\right)}{r_{\ell}\left(\tau_{\ell}\right)} d s \\
& =\int_{0}^{t} G(s) \frac{\hat{r}_{\ell}(\tau, s)}{\bar{r}_{\ell}(\tau)} \frac{f_{\ell}\left(\tau_{\ell}+s\right)}{r_{\ell}\left(\tau_{\ell}\right)} d s
\end{aligned}
$$

and that $\Phi(t)=\hat{\Phi}_{\ell}\left(t-t_{1}\right)\left(J_{\ell} e^{A t_{1}}\right)$ when the transition $\ell \in \mathcal{L}$ is first triggered, where $\hat{\Phi}_{\ell}\left(t-t_{1}\right)$ is the transition matrix from $t_{1}$ to $t$ starting the process at $\pi_{\ell}^{0}\left(\tau+s 1_{n_{\ell}}\right)$ where $\pi_{\ell}^{0}$ is defined by (20). Thus

$$
\begin{aligned}
& \mathbb{E}_{\tau}\left[\Phi(t)^{\top} Y(v(t)) \Phi(t) \mathbf{1}_{C_{\ell}(t)}\right]=\int_{0}^{t}\left(J_{\ell} e^{A s}\right)^{\top} \ldots \\
& \mathbb{E}_{\pi_{\ell}^{0}\left(\tau+s 1_{n_{\ell}}\right)}\left[\hat{\Phi}(t-s)^{\top} Y(v(t-s)) \hat{\Phi}(t-s)\right] J_{\ell} e^{A s} \alpha_{\ell}(\tau, s) d s,
\end{aligned}
$$

where $\alpha_{\ell}(\tau, s):=\frac{\hat{r}_{\ell}(\tau, s)}{\bar{r}_{\ell}(\tau)} \frac{f_{\ell}\left(\tau_{\ell}+s\right)}{r_{\ell}\left(\tau_{\ell}\right)}$. By construction of the process

$$
\begin{aligned}
\mathbb{E}_{\pi_{\ell}^{0}\left(\tau+s 1_{n_{\ell}}\right)}\left[\left(\hat{\Phi}_{\ell}(t-s)^{\top} Y(v(t-s)) \hat{\Phi}_{\ell}(t-s)\right]=\right. \\
\mathbb{E}_{\pi_{\ell}^{0}\left(\tau+s 1_{n_{\ell}}\right)}\left[\Phi(t-s)^{\top} Y(v(t-s)) \Phi(t-s)\right] .
\end{aligned}
$$

Replacing (53) in (52), (52) in (51), and (51) in (43) we obtain

$X_{1}(\tau)=\sum_{\ell=1}^{n_{\ell}} \hat{X}_{1}^{\ell}(\tau)+\int_{0}^{\bar{\gamma}} e^{A^{\top} t} Y\left(\tau+t 1_{n_{\ell}}\right) e^{A t} \Pi_{\ell=1}^{n_{\ell}} \frac{r_{\ell}\left(\tau_{\ell}+t\right)}{r_{\ell}\left(\tau_{\ell}\right)} d t$

where

$$
\begin{array}{r}
\hat{X}_{1}^{\ell}(\tau)=\int_{0}^{\infty} \int_{0}^{t} \mathbb{E}_{\pi_{\ell}^{0}\left(\tau+s 1_{n_{\ell}}\right)}\left[( J _ { \ell } e ^ { A s } ) ^ { \top } \left(\Phi(t-s)^{\top} Y(v(t-s)) \ldots\right.\right. \\
\left.\Phi(t-s)\left(J_{\ell} e^{A s}\right) \alpha_{\ell}(\tau, s)\right] d s d t
\end{array}
$$

Changing the order of integration in the latter expression we have that (54) can be written as

$$
\begin{aligned}
& X_{1}(\tau)=\int_{0}^{\bar{\gamma}} e^{A^{\top} t} Y\left(\tau+s 1_{n_{\ell}}\right) e^{A t} \Pi_{\ell=1}^{n_{\ell}} \frac{r_{\ell}\left(\tau_{\ell}+t\right)}{r_{\ell}\left(\tau_{\ell}\right)} d t+ \\
& \sum_{\ell=1}^{n_{\ell}} \int_{0}^{\bar{\gamma}}\left(J_{\ell} e^{A s}\right)^{\top} X_{1}\left(\pi_{\ell}^{0}\left(\tau+s 1_{n_{\ell}}\right)\right) J_{\ell} e \frac{\hat{r}_{\ell}(\tau, s)}{\bar{r}_{\ell}\left(\tau_{\ell}\right)} \frac{f_{\ell}\left(\tau_{\ell}+s\right)}{r_{\ell}\left(\tau_{\ell}\right)} d s
\end{aligned}
$$

With similar computations one can conclude that

$$
\begin{aligned}
& X_{2}(\tau)=\sum_{\ell=1}^{n_{\ell}} \int_{0}^{\bar{\gamma}} e^{A^{\top} s} Z\left(\tau+s 1_{n_{\ell}}\right) e^{A s} \frac{\hat{r}_{\ell}(\tau, s)}{\bar{r}_{\ell}(\tau)} \frac{f_{l}\left(\tau_{\ell}+s\right)}{r_{\ell}\left(\tau_{\ell}\right)} d s+ \\
& \sum_{\ell=1}^{n_{\ell}} \int_{0}^{\bar{\gamma}}\left(J_{\ell} e^{A s}\right)^{\top} X_{2}\left(\pi_{\ell}^{0}\left(\tau+s 1_{n_{\ell}}\right)\right) J_{\ell} e^{A s} \frac{\hat{r}_{\ell}(\tau, s)}{\bar{r}_{\ell}(\tau)} \frac{f_{\ell}\left(\tau_{\ell}+s\right)}{r_{\ell}\left(\tau_{\ell}\right)} d s
\end{aligned}
$$

Since $X(\tau)=X_{1}(\tau)+X_{2}(\tau)$ adding $X_{1}(\tau)$ and $X_{2}(\tau)$ we obtain $(50)$.

Conversely, suppose that there exists a solution $P \in \mathcal{V}$ to (47). Then one can verify that

$$
\begin{aligned}
& X(\tau)=W(\tau)+ \\
& \sum_{\ell=1}^{n_{\ell}} \int_{0}^{\gamma_{\ell}}\left(J_{\ell} e^{A s}\right)^{\top} P_{\ell}\left(\hat{\pi}_{\ell}(\tau)+s 1_{n_{\ell-1}}\right) J_{\ell} e^{A s} \frac{\hat{r}_{\ell}(\tau, s)}{\bar{r}_{\ell}(\tau)} \frac{f_{\ell}\left(\tau_{\ell}+s\right)}{r_{\ell}\left(\tau_{\ell}\right)} d s
\end{aligned}
$$

satisfies all the assumptions of the function $X(\tau)$ of Theorem 10, and therefore (27) is MES. In fact, if there exists a solution $P \in \mathcal{V}$ to (47) one can obtain an explicit expression for the solution to (47) (cf. Theorem 12), which is given by

$$
P=\sum_{i=0}^{\infty} \mathfrak{L}^{i}(U)
$$

where $\mathfrak{L}^{i}$ denotes the composite operator obtained by applying $i$ times $\mathfrak{L}$, e.g., $\mathfrak{L}^{2}(P)=\mathfrak{L}(\mathfrak{L}(P))$ and $\mathfrak{L}^{0}(P):=$ $P$. From (56) we can conclude that $P$ is bounded and differentiable with respect to $\tau$, since we assume that the $f_{\ell}$ are differentiable. Then, it is clear that $X(\tau)$ is bounded, $X(\tau) \geq W(\tau) \geq c_{1} I, \forall_{\tau \in B}$, for some $c_{1}>0$ and (40) can be obtained by direct computation.

\section{(iii) Positive solution of the Fredholm equation}

As a prelude to the next result, we note that $\mathcal{U}$ is a cone in the Hilbert space (and hence Banach space, with the usual norm inherited by the inner product) $\mathcal{P}$, in the sense of [17] since (i) it is closed; (ii) if $U, W \in \mathcal{U}$ then $\alpha_{1} U+\alpha_{2} W \in \mathcal{U}$ for $\alpha_{1} \geq 0$ and $\alpha_{2} \geq 0$; and (iii) the set $^{4}-\mathcal{U}:=\{-P: P \in \mathcal{U}\}$ intersects $\mathcal{U}$ only at the zero vector. Moreover, this cone is reproducing in $\mathcal{P}$, i.e., if $Z \in \mathcal{P}$, then there exists $U, W \in \mathcal{U}$ such that $Z=U-W$ (take for example, $U_{i}(\tau)=Z_{i}(\tau)+\epsilon I$ and $W_{i}(\tau)=\epsilon I$ for sufficiently large $\epsilon>0$ such that $P_{i}(\tau)+\epsilon I>0$ for all $\left.i \in\left\{1, \ldots, n_{\ell}\right\}, \tau \in B\right)$. The operator $\mathfrak{L}$ is a positive operator with respect to $\mathcal{U}$, i.e., $\mathfrak{L}(U) \in \mathcal{U}$ if $U \in \mathcal{U}$.

Theorem 12 The equation (47) has a solution $P \in \mathcal{V}$ if and only if $r_{\sigma}(\mathfrak{L})<1$.

Note that the main result, Theorem 4, can be concluded from Theorems 11, 12.

Proof Sufficiency follows from the fact that if $r_{\sigma}(\mathfrak{L})<1$ then $P=\sum_{i=0}^{\infty} \mathfrak{L}^{i}(U)$ exists which is the solution to

\footnotetext{
${ }^{4}$ Recall that addition and multiplications by scalar are defined in a natural way in $\mathcal{P}$, e.g. if $P=\left(P_{1}, \ldots, P_{n_{\ell}}\right) \in \mathcal{P}$ then $\left.-P:=\left(-P_{1}, \ldots,-P_{n_{\ell}}\right)\right\}$
} 
$P=\mathfrak{L}(P)+U$. Since $\mathfrak{L}$ is a positive operator with respect to $\mathcal{U}, P$ is a summation of $U \in \mathcal{V}$ plus elements in $\mathcal{U}$. Thus, taking into account the definitions of $\mathcal{U}$ and $\mathcal{V}$, we conclude that $P$ belongs to $\mathcal{V}$.

To prove necessity, we start by noticing that it is possible to prove that the dual cone (cf. $[6, \text { Sec. 2.6 }]^{5}$ ) of $\mathcal{U}$ can be identified with itself, i.e., using the nomenclature of $[6$, Sec. 2.6], $\mathcal{U}$ is self-dual. The proof follows similar arguments used to prove that the cone of positive semidefinite matrices is self-dual (cf. [6, p.52]), and is therefore omitted. From [17, p.22, Th. 2.5], we conclude that the adjoint operator $\mathfrak{L}^{*}$ is also a positive operator with respect to $\mathcal{U}$, and using [17, Th. 9.2] which states that a completely continuous positive operator with respect to a reproducing cone has an eigenvalue that equals the spectral radius and an eigenvector that belongs to the solid cone, we conclude that there exists $W \in \mathcal{U}$ (other than the zero element) such that

$$
\mathfrak{L}^{*}(W)=r_{\sigma}\left(\mathfrak{L}^{*}\right) W
$$

In fact, $\mathfrak{L}^{*}$ is a compact operator, since $\mathfrak{L}$ is a compact operator (cf. [9, p.178]), which can be concluded from [8, p. 165, Th. 4.1], and a compact operator in a Banach space is completely continuous (cf. [9, p.177]). Suppose that $r_{\sigma}(\mathfrak{L}) \geq 1$ and $(47)$ has a solution $P \in \mathcal{V}$. Then $r_{\sigma}\left(\mathfrak{L}^{*}\right)=r_{\sigma}(\mathfrak{L}) \geq 1$. Taking the inner product on both sides of (47) with $W \in \mathcal{U}$, such that (57) holds, yields

$$
\begin{aligned}
& \langle W, P\rangle=\langle W, \mathfrak{L}(P)\rangle+\langle W, U\rangle \Leftrightarrow \\
& \langle W, P\rangle=\left\langle\mathfrak{L}^{*}(W), P\right\rangle+\langle W, U\rangle \Leftrightarrow \\
& \langle W, P\rangle\left(1-r_{\sigma}\left(\mathfrak{L}^{*}\right)\right)=\langle W, U\rangle
\end{aligned}
$$

Now $\langle W, P\rangle \geq 0$, since $W, P \in \mathcal{U}$. Moreover, one can conclude that $\langle W, U\rangle>0$, since $W$ is different from the zero element in $\mathcal{U}$ and one can conclude from (48) that $U \in \mathcal{V}$. Thus, from (58) we conclude that $r_{\sigma}\left(\mathfrak{L}^{*}\right)=$ $r_{\sigma}(\mathfrak{L}) \geq 1$ leads to a contradiction.

\section{Batch Reactor}

This example considers the control of a linearized model of an open loop unstable two-input two-output batch reactor, controlled by a PI controller. It is a widely used example in networked control (see,e.g., [24], [14]). The plant and controller take the form (2), and (3), with $f_{P}\left(x_{P}, \hat{u}\right)=A_{P} x_{P}+B_{P} \hat{u}, g\left(x_{P}\right)=C_{P} x_{P}$, and $f_{C}\left(x_{C}, \hat{y}\right)=A_{C} x_{C}+B_{C} \hat{y}, h\left(x_{C}, \hat{y}\right)=C_{C} x_{C}+D_{C} \hat{y}$. The expressions for $\left(A_{P}, B_{P}, C_{P}\right)$ and $\left(A_{C}, B_{C}, C_{C}, D_{C}\right)$ can be found in [2]. The actuator is directly connected $\hat{u}(t)=$

\footnotetext{
5 The nomenclature used in [17, Ch. 2] is adjoint cone instead of dual cone
}

$u(t)$. However, the sensors are linked to the plant through communications networks.

In [2], it is assumed that the outputs are sent in a roundrobin fashion through a single shared communication network. When the distribution of the intervals between consecutive transmissions is assumed to be, e.g., uniform with a support $\gamma$, we can use the results in [2] to study the stability of this system.

Suppose now that, instead of transmitting the two measurements in a round robin fashion through the same communication network, the two sensors transmit data through two independent communication links. We assume that both links are shared with other users and that the intervals between consecutive transmissions can be modeled by independent processes with support in the interval $\left[0, \gamma_{\ell}\right]$ for the link associated with the output $y_{\ell}, \ell \in\{1,2\}$. We can cast this system in the framework of Section 2, and use the techniques developed in this paper to study the stability in this latter case.

When two links are used to transmit the measurements of the two sensors, we can use Theorem 4 to investigate the stability of the system as a function of the distributions for the intersampling times on each network. The results obtained are summarized in Figure 2 for the case of uniform distributions with different supports. If the distributions of the two links have the same support then stability is preserved for every $\gamma_{1}, \gamma_{2} \in[0, \bar{\gamma}]$, with $\bar{\gamma}=0.18$. It is interesting to compare this with the case of a round-robin single-link protocol where it was shown in [2] that the maximum support of a uniform distribution for which stability could be guaranteed was $\gamma=0.11$. With a round-robin protocol, this would lead to a distribution between consecutive samples for the same sensor that is triangular with support 0.22 . However, note that in this case the duration of the intervals between transmissions of the two outputs are not independent, and a different approach must be used to assert stability (see [2]). If the two links have different supports one can conclude from the Figure 2 that the mean exponential stability of the closed-loop is lost for a lower value of the support of the distributions associated with the output $y_{2}$ than the value of the support associated with the output $y_{1}$.

\section{Final Remarks and Future Work}

We provided several stability results for impulsive systems with several reset maps triggering asynchronously at independent and identically distributed spaced times, motivated by their applications to networked control systems. Since our main focus was to capture the asynchronous nature of the resets corresponding to transmissions in independent networks, we considered several assumptions for simplicity. We point out here some directions to drop three of these assumptions. First, we 


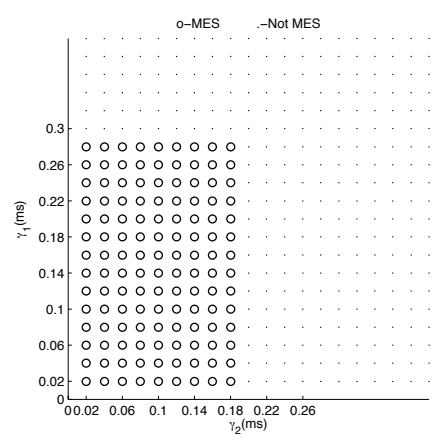

Fig. 2. MES for various values of the support of a uniform distributions of the transmission intervals of two independent links.

considered finite supports for the probability distributions that model the intervals between resets, except in the special case where these distributions are exponential. In a previous work [4], we provided necessary and sufficient stability conditions for the case in which the dynamic and reset maps are linear, there is only one reset map $\left(n_{\ell}=1\right)$, and the supports may be unbounded, using an approach based on Volterra equations, which does not appear to generalize for $n_{\ell}>1$. As in [4], and as in the case of exponential distributions considered in the present paper, besides the stability condition provided in Theorem 4, other conditions are required that take into account inter-jump behavior (condition (37) in the case of exponential distributions). However, it is not clear to the authors how to obtain necessary and sufficient conditions for stability (in a stochastic sense) as in [4] for the case of unbounded support when $n_{\ell}>1$. Second, we considered no packet drops in the networked control setup and we assumed that no two nodes share the same network, in which case a protocol such as round-robin would be required. Taking into account these features would entail considering stochastic transitions to model packet drops and including auxiliary discrete modes to model the protocol (cf., e.g., [3]). Since piecewise deterministic processes allow to take into account discrete modes and stochastic rest maps, it is reasonable to believe that one can take these features into account, generalizing the results in the present paper to this case. Third, we considered no disturbances acting on the plant. Piecewise deterministic processes do not allow to model for example stochastic disturbances between resets. To capture this, [15] considers a so-called model jump diffusions with state-dependent intensities. Combining the ideas presented here and in [15] can therefore be a direction for future work to capture stochastic disturbances in the model. Another possible direction for future work is to exploit in-network processing schemes [7], [21] to enhance the stability properties and performance of the networked control systems considered in the present paper.

\section{Appendix}

Proof of Theorems 1 and 3

We start by describing a construction for the process $\mathbf{x}$, described by (18), which mimics the construction of a piecewise deterministic process, as described in [10, p. 59]. Let $\Omega:=\left\{u_{k}^{1}, u_{k}^{2}, k \geq 0\right\}$ where $\left\{u_{k}^{1}, k \geq 0\right\}$ and $\left\{u_{k}^{2}, k \geq 0\right\}$ are mutually independent and identically distributed random variables uniformly distributed in the interval $[0,1]$. Let also $\phi_{x}\left(s, x\left(t_{k}\right)\right), \phi_{v}\left(s, v\left(t_{k}\right)\right)$ be the flows at time $s$ of the systems described by $\dot{x}(t)=$ $a(x(t))$ and $\dot{v}(t)=1$ with initial conditions $x\left(t_{k}\right)$ and $v\left(t_{k}\right)$, respectively. Note that $\phi_{v}\left(s, v\left(t_{k}\right)\right)=v\left(t_{k}\right)+s 1_{n_{\ell}}$. Set $k=0$ and $t_{0}=0, \mathbf{x}\left(t_{0}\right)=\left(x_{0}, \tau\right)$, and consider the process $\mathbf{x}(t)=(x(t), v(t))$ obtained by iteratively repeating:

(I) Obtain $h_{k}$ from

$$
h_{k}=\inf \left\{t: e^{-\int_{0}^{t} \lambda_{T}\left(\phi_{v}\left(s, v\left(t_{k}\right)\right)\right) d s} \leq u_{k}^{1}\right\} .
$$

where $\lambda_{T}$ is described by (45). Set $t_{k+1}=t_{k}+h_{k}$, and for $t \in\left[t_{k}, t_{k+1}\right)$ make

$$
\mathbf{x}(t)=\left(\phi_{x}\left(t-t_{k}, x\left(t_{k}\right)\right), \phi_{v}\left(t-t_{k}, v\left(t_{k}\right)\right)\right) .
$$

(II) Make $\mathbf{x}\left(t_{k+1}\right)=\psi\left(u_{k}^{2}, \mathbf{x}\left(t_{k+1}^{-}\right)\right)$, where

$\psi(w,(x, \tau))=\left(j_{\ell}(x), \pi_{\ell}^{0}(\tau)\right) \chi_{w \in\left(\sum_{j=1}^{\ell-1} \frac{\lambda_{j}\left(\tau_{j}\right)}{\lambda_{T}(\tau)}, \sum_{j=1}^{\ell} \frac{\lambda_{j}\left(\tau_{j}\right)}{\lambda_{T}(\tau)}\right]}$,

and $\chi_{x \in A}$ denotes the characteristic function, i.e.,

$$
\chi_{w \in A}=\left\{\begin{array}{l}
1 \text { if } w \in A \\
0, \text { if } w \notin A
\end{array} .\right.
$$

Remark 13 Note that (59) simply states that

$$
\operatorname{Prob}\left[h_{k}>s \mid \mathbf{x}\left(t_{k}\right)\right]=\Pi_{j=1}^{n_{\ell}} r_{j}\left(s+v_{j}\left(t_{k}\right)\right), \quad \forall_{k \geq 0}
$$

and (61) simply states that

$\operatorname{Prob}\left[\mathbf{x}\left(t_{k}\right)=\left(j_{\ell}\left(x\left(t_{k}^{-}\right)\right), \pi_{\ell}^{0}\left(v\left(t_{k}^{-}\right)\right)\right) \mid \mathbf{x}\left(t_{k}^{-}\right)\right]=\frac{\lambda_{\ell}\left(\tau_{j}\left(t_{k}^{-}\right)\right)}{\lambda_{T}\left(\tau\left(t_{k}^{-}\right)\right)}$.

We choose to use the description (I) and (II) to mimic the piecewise deterministic process construction in [10, p. 59], which allows us to use the results from [10].

The next Theorem establishes the connection between (1), (16), and piecewise deterministic processes. 
Theorem 14 The stochastic process $(x(t), v(t))$, described by (1) and (16), can be realized in the probability space $\Omega$ and constructed as the piecewise deterministic process defined by steps (I) and (II).

Proof For the process $(x(t), v(t))$, described by (1), (16), define $\left\{t_{k} \geq 0\right\}$ with $t_{k}<t_{k+1}, \forall_{k \geq 0}$ as a set containing the union of all the jump times in (1), i.e., $\left\{t_{k} \geq 0\right\}=$ $\cup_{\ell=1}^{n_{\ell}}\left\{t_{r_{\ell}}^{\ell}, r_{\ell} \geq 0\right\}$, let $\left\{h_{k}:=t_{k+1}-t_{k}, k \geq 0\right\}, h_{-1}:=0$, and consider the following discrete-time process

$$
z_{k}:=\left(h_{k-1}, x\left(t_{k}\right), v\left(t_{k}\right)\right)
$$

There exist a one to one relation between $z_{k}$ and $\mathbf{x}(t)$, described by (1), and (16). In fact, given a sample path $(x(t), v(t))$ one can identify the jump times $t_{k}$ by the times at which $v_{\ell}\left(t_{k}\right)=0$ for some $\ell$, and from these construct $h_{k-1}$ and hence $z_{k}$. Conversely, from $z_{k}$ we can obtain $h_{k}$ and hence $t_{k}$, and construct $(x(t), \tau(t))$ from $\left(x\left(t_{k}\right),\left(v\left(t_{k}\right)\right)\right.$ as

$$
\begin{array}{r}
(x(t), v(t))=\left(\phi_{x}\left(t-t_{k}, x\left(t_{k}\right)\right), \phi_{v}\left(t-t_{k}, v\left(t_{k}\right)\right)\right), \\
t_{k} \leq t<t_{k+1} .
\end{array}
$$

Moreover, $z_{k}$ is a discrete-time Markov process. To see this, it suffices to prove that

$\operatorname{Prob}\left(z_{k+1} \in D \mid z_{r}, 0 \leq r \leq k\right)=\operatorname{Prob}\left(z_{k+1} \in D \mid z_{k}\right), \forall_{k \geq 0}$,

where $D$ is a measurable set. Since at $t_{k}$, a time $\tau_{\ell}\left(t_{k}\right)$ has elapsed since the last jump associated with the reset map $\ell \in \mathcal{L}$, the time $t_{k+1}$ equals $t_{k+1}=t_{k}+h_{k}$, where

$$
h_{k}:=\min _{\ell \in\left\{1, \ldots, n_{\ell}\right\}}\left\{w_{k}^{\ell}\right\}
$$

and $w_{k}^{\ell}$ are random variables such that $\operatorname{Prob}\left(w_{k}^{\ell}>s\right)=$ $\frac{r_{\ell}\left(v_{\ell}\left(t_{k}\right)+s\right)}{r_{\ell}\left(v_{\ell}\left(t_{k}\right)\right)}$, where the $r_{\ell}$ are described by (12). Thus,

$$
\operatorname{Prob}\left(h_{k+1}>s \mid z_{r}, 0 \leq r \leq k\right)=\Pi_{\ell=1}^{n_{\ell}} \frac{r_{\ell}\left(v_{\ell}\left(t_{k}\right)+s\right)}{r_{\ell}\left(v_{\ell}\left(t_{k}\right)\right)},
$$

Let $\xi_{k+1}$ denote which reset map triggers at $t_{k+1}$, i.e.,

$$
\xi_{k+1}:=\operatorname{argmin}_{\ell \in\left\{1, \ldots, n_{\ell}\right\}}\left\{w_{k}^{\ell}\right\} .
$$

Then,

$$
\begin{aligned}
& \operatorname{Prob}\left[\xi_{k+1}=\ell \mid h_{k} \in[s, s+\epsilon) \wedge z_{r}, 0 \leq r \leq k\right]= \\
& \quad \frac{\operatorname{Prob}\left[h_{k} \in[s, s+\epsilon) \wedge \xi_{k+1}=\ell \mid z_{r}, 0 \leq r \leq k\right]}{\sum_{j=1}^{n_{\ell}} \operatorname{Prob}\left[h_{k} \in[s, s+\epsilon) \wedge \xi_{k+1}=j \mid z_{r}, 0 \leq r \leq k\right]}
\end{aligned} .
$$

Now,

$$
\begin{aligned}
& \operatorname{Prob}\left[h_{k} \in[s, s+\epsilon) \wedge \xi_{k+1}=\ell \mid z_{r}, 0 \leq r \leq k\right] \\
& =\operatorname{Prob}\left[w_{k}^{\ell} \in[s, s+\epsilon) \wedge w_{k}^{j}>w_{k}^{\ell}, \forall_{j \neq \ell} \mid z_{r}, 0 \leq r \leq k\right] \\
& =\int_{s}^{s+\epsilon} \Pi_{j=1, j \neq \ell}^{n_{\ell}} \frac{r_{j}\left(v_{j}\left(t_{k}\right)+q\right)}{r_{j}\left(v_{j}\left(t_{k}\right)\right)} \frac{f_{\ell}\left(v_{\ell}\left(t_{k}\right)+q\right)}{r_{\ell}\left(v_{\ell}\left(t_{k}\right)\right)} d q
\end{aligned}
$$

Replacing (68) in (67), taking the limit as $\epsilon \rightarrow 0$, and dividing the numerator and denominator of the right hand side of $(67)$ by $\prod_{j=1}^{n_{\ell}} r_{j}\left(v_{j}\left(t_{k}+s\right)\right)$, we obtain

$$
\begin{aligned}
\operatorname{Prob}\left[\xi_{k+1}\right. & \left.=\ell \mid h_{k}=s \wedge z_{r}, 0 \leq r \leq k\right] \\
& =\frac{\lambda_{\ell}\left(v_{\ell}\left(t_{k}\right)+s\right)}{\lambda_{T}\left(v\left(t_{k}\right)+s 1_{n_{\ell}}\right)}=\frac{\lambda_{\ell}\left(v_{\ell}\left(t_{k+1}^{-}\right)\right)}{\lambda_{T}\left(v\left(t_{k+1}^{-}\right)\right)} .
\end{aligned}
$$

where $\lambda_{T}\left(v\left(t_{k+1}^{-}\right)\right):=\sum_{\ell=1}^{n_{\ell}} \lambda_{\ell}\left(v_{\ell}\left(t_{k+1}^{-}\right)\right)$. Thus, we conclude that

$$
\begin{gathered}
\operatorname{Prob}\left[\left(x\left(t_{k}\right), v\left(t_{k}\right)\right)=\left(j_{\ell}\left(x\left(t_{k}^{-}\right)\right), \pi_{\ell}^{0}\left(v\left(t_{k}^{-}\right)\right)\right) \mid h_{k} \wedge z_{r}, 0 \leq r \leq k\right] \\
=\frac{\lambda_{\ell}\left(v_{\ell}\left(t_{k}^{-}\right)\right)}{\lambda_{T}\left(v\left(t_{k}^{-}\right)\right)}
\end{gathered}
$$

From (69), and (66), we conclude that

$$
\begin{gathered}
\operatorname{Prob}\left[h_{k} \in[c, d], x\left(t_{k+1}\right) \in E_{x}, v\left(t_{k+1}\right) \in E_{v} \mid z_{r}, 0 \leq r \leq k\right] \\
=\sum_{\ell=1}^{n_{\ell}}\left[\int_{c}^{d} \chi_{j_{\ell}\left(\phi_{x}\left(s, \mathbf{x}\left(t_{k}\right)\right)\right) \in E_{x} \wedge \phi_{v}\left(s, \mathbf{x}\left(t_{k}\right)\right) \in E_{v} \cdots}\right. \\
\left.\left(\Pi_{j=1, j \neq \ell}^{n_{\ell}} \frac{r_{j}\left(v_{j}\left(t_{k}\right)+s\right)}{r_{j}\left(v_{j}\left(t_{k}\right)\right)}\right) \frac{f_{\ell}\left(v_{\ell}\left(t_{k}\right)+s\right)}{r_{\ell}\left(v_{\ell}\left(t_{k}\right)\right)}\right] d s,
\end{gathered}
$$

where $\chi_{(\ldots)}$ denotes the characteristic function (62). Note that (70) depends only on $z_{k}=\left(h_{k-1}, x\left(t_{k}\right), v\left(t_{k}\right)\right)$ and thus the Markov property (65) holds.

Consider now the piecewise deterministic process defined by steps (I) and (II) and let

$$
y_{k}:=\left(h_{k-1}, x\left(t_{k}\right), v\left(t_{k}\right)\right)
$$

where $h_{-1}=0$, and $h_{k-1}, t_{k}, x\left(t_{k}\right), v\left(t_{k}\right)$ are now the variables defined in steps (I) and (II). Then, by construction of the process and Remark 13, we immediately obtain that (66), and (69), also hold for this process, and this implies that (70) also holds for this process. Thus $y_{k}$ is a Markov process with the same transition probability function, i.e., an alternative realization to $z_{k}$. Since there is a one-to-one relation between $z_{k}$ and the process defined by (1), (16), and there is a one-to-one relation between $y_{k}$ and the process defined by the piecewise deterministic process construction described by steps (I) and (II), and both processes are completed in the same way between jump times (see (60), (64)), we conclude that the process (1), (16) can be constructed as the piecewise deterministic process specified by steps (I) and (II). 
Proof (of Theorem 1) Theorem 14 allows us to apply the results available in [10]. In particular, Theorem 1 follows directly from $[10$, p.33, (14.17)], [10, p.66, Th. (26.14)] and [10, p. 70, Rem. (26.16)]), provided that we can prove that the assumption in [10, p.60, (24.4)] that the expected value of the number of jumps up to a given time $t$ is bounded, which when specialized to the stochastic process $(x(t), v(t))$, described by (1), is equivalent to saying that $\mathbb{E}\left[\sum_{\ell=1}^{n_{\ell}} N_{\ell}(t)\right]<\infty$, where

$$
N_{\ell}(t):=\max \left\{k \in \mathbb{N}: t_{k}^{\ell} \leq t\right\} .
$$

This is in fact true, since each $N_{\ell}(t)$ is a renewal process [22] with intervals between renewal times following a probability density function $f_{\ell}$ with no atom points, and therefore $\mathbb{E}\left[N_{\ell}(t)\right]<\infty$ (cf. $[22$, p. 186]). From this latter fact, one can also conclude that there is zero probability of an infinite number of jumps occurring in finite time (cf. [22, p. 186]), which precludes zeno phenomenon (cf. [12]).

Proof (of Theorem 3) To prove sufficiency, we use Theorem 1 applied to the function

$$
W(\mathbf{x}(t), t):=e^{r_{1} t} V(\mathbf{x}(t))
$$

where $V$ is a positive function, which equals zero at zero, and satisfies (25) and (26), $r_{1}$ is a positive constant such that $r_{1}>\frac{r}{c_{2}}$, and it is implicit that the process $(\mathbf{x}(t), t)$ is a piecewise deterministic process if $\mathbf{x}(t)$ is a piecewise deterministic process (cf. [10, p.84]). We need to show that $W$ satisfies (21). Since we assume that $a$ and $j_{\ell}$ are globally Lipschitz, we have $\|a(x)\| \leq L_{1}\|x\|, \forall_{x \in \mathbb{R}^{n}}$ and for every $\ell \in \mathcal{L}$, we have $\left\|j_{\ell}(x)\right\| \leq L_{2}\|x\|, \forall_{x \in \mathbb{R}^{n}}$. Thus, between jump times,

$$
\|x(t)\|^{2} \leq e^{2 L_{1}\left(t-t_{k}^{\ell}\right)}\left\|x\left(t_{k}^{\ell}\right)\right\|^{2}, t \in\left[t_{k}^{\ell}, t_{k+1}^{\ell}\right),
$$

(cf. [16, p.107, Exercise 3.17]) and at jump times, $\left\|x\left(t_{k}^{\ell}\right)\right\|^{2} \leq\left(L_{2}\right)^{2}\left\|x\left(t_{k}^{\ell-}\right)\right\|^{2}$. Thus,

$$
\mathbb{E}\left[\|x(t)\|^{2}\right] \leq \mathbb{E}\left[e^{2 L_{1} t} \Pi_{\ell=1}^{n_{\ell}}\left(L_{2}\right)^{2 N_{\ell}(t)}\right]\left\|x_{0}\right\|^{2}
$$

where $N_{\ell}(t)$ is the number of jumps associated with the reset map $\ell$ up to the time $t$, described by (71). We also have

$$
\begin{aligned}
& \mathbb{E}\left[\sum_{t_{k}^{\ell} \leq n}\left|W\left(\mathbf{x}\left(t_{k}^{\ell}\right)\right)-W\left(\mathbf{x}\left(t_{k}^{\ell-}\right)\right)\right|\right] \\
& \left.\quad \leq \mathbb{E}\left[\sum_{t_{k}^{\ell} \leq n} c_{2} e^{r_{1} n} \| x\left(t_{k}^{\ell}\right)\right) \|^{2}\right] \\
& \quad \leq c_{2} e^{2 L_{1} n} e^{r_{1} n}\left\|x_{0}\right\|^{2} \sum_{j=1}^{n_{\ell}} \sum_{k=0}^{\infty}\left(L_{2}\right)^{2 k} \mathbb{E}\left[\chi_{t_{k}^{\ell} \leq n}\right]
\end{aligned}
$$

where we used (25), and (72), and $\chi_{(\ldots)}$ denotes the characteristic function (62). Note that $\mathbb{E}\left[\chi_{t_{k}^{\ell} \leq n}\right]=$ $\operatorname{Prob}\left[N_{\ell}(n) \geq k\right]$. The fact that the right-hand side of (73) is bounded is a direct application of [22, p.186, Th. 3.3.1], and therefore $W$ satisfies (21).

From Theorem 1

$$
\mathbb{E}(W(\mathbf{x}(t), t))=W(\mathbf{x}, 0)+\mathbb{E}\left[\int_{0}^{t} \mathfrak{A} W(\mathbf{x}(s), s) d s\right]
$$

for an initial condition $\mathrm{x}=\left(x_{0}, \tau\right)$. From [10, p. 84], we can conclude that

$$
\mathfrak{A} W(\mathbf{x}(s), s)=r_{1} W(\mathbf{x}(s), s)+e^{r_{1} s} \mathfrak{A} V(\mathbf{x}(s))
$$

and using (26) we obtain

$$
\begin{aligned}
& \mathbb{E}(W(\mathbf{x}(t), t)) \leq W(\mathbf{x}, 0)+ \\
& \mathbb{E}\left[\int_{0}^{t} r_{1} W(\mathbf{x}(s), s)-r e^{r_{1} s}\|x(s)\| d s\right]
\end{aligned}
$$

Using (25) and interchanging expectation with integral operations, we obtain

$$
\mathbb{E}(W(\mathbf{x}(t), t)) \leq W(\mathbf{x}, 0)+\left(r_{1}-\frac{r}{c_{2}}\right) \int_{0}^{t} \mathbb{E}[W(\mathbf{x}(s), s)] d s .
$$

which implies, from the integral form of the Gronwall's inequality [5, Lemma 1], that

$$
\begin{aligned}
& \mathbb{E}\left[V(\mathbf{x}(t)) e^{r_{1} t}\right]=\mathbb{E}[W(\mathbf{x}(t), t)] \\
& \leq \mathbb{E}[W(\mathbf{x}, 0)] e^{\left(r_{1}-\frac{r}{c_{2}}\right) t}=V(\mathbf{x}) e^{\left(r_{1}-\frac{r}{c_{2}}\right) t} .
\end{aligned}
$$

Note that we can apply the Gronwall's inequality since $r_{1}-\frac{r}{c_{2}}>0$, and this is the reason to work with $W$, instead of directly using $V$. From (74), we conclude that

$$
\mathbb{E}[V(\mathbf{x}(t))] \leq V(\mathbf{x}) e^{-\frac{r}{c_{2}} t}
$$

and using (25), we conclude that:

$$
\begin{aligned}
\mathbb{E}\left[\|x(t)\|^{2}\right] \leq \frac{1}{c_{1}} \mathbb{E}[V(\mathbf{x}(t))] \leq \frac{1}{c_{1}} & V(\mathrm{x}) e^{-\frac{r}{c_{2}} t} \\
& \leq \frac{c_{2}}{c_{1}}\left\|x_{0}\right\|^{2} e^{-\frac{r}{c_{2}} t} .
\end{aligned}
$$

Necessity is obtained by proving that

$$
V(\mathrm{x}):=V_{1}(\mathrm{x})+V_{2}(\mathrm{x})
$$


satisfies (25) and (26) where

$$
\begin{aligned}
V_{1}(\mathrm{x}) & :=\int_{0}^{+\infty} \mathbb{E}_{\mathrm{x}}\left[x(s)^{\top} Y(v(s)) x(s)\right] d s \\
V_{2}(\mathrm{x}) & :=\sum_{\ell=1}^{n_{\ell}} \sum_{k>0} \mathbb{E}_{\mathrm{x}}\left[x\left(t_{k}^{\ell-}\right)^{\top} Z\left(v\left(t_{k}^{\ell-}\right)\right) x\left(t_{k}^{\ell-}\right)\right],
\end{aligned}
$$

$\mathbb{E}_{\mathrm{x}}$ emphasizes that expectation subsumes that the process starts at an initial condition $\mathrm{x}=\left(x_{0}, \tau\right)$, and $a_{1} \leq$ $Y(\tau) \leq a_{2} I, \forall \tau \in B$, and $b_{1} \leq Z(\tau) \leq b_{2} I, \forall \tau \in B$, are differentiable functions, where $a_{i}, b_{i}, i \in\{1,2\}$ are positive constants.

To see that $V(\mathrm{x}) \geq c_{1}\left\|x_{0}\right\|^{2}$ denote by $t_{1}$ the time of the first jump, which can be from any of the $n_{\ell}$ reset maps, and note that $V(\mathrm{x}) \geq a_{1} \int_{0}^{t_{1}} \mathbb{E}_{\mathrm{x}}\left[x(s)^{\top} x(s)\right] d s+$ $b_{1} \mathbb{E}_{\mathbf{x}}\left[x\left(t_{1}^{-}\right)^{\top} x\left(t_{1}^{-}\right)\right]$for any $t_{1} \geq 0$. Let $L_{1}$ be a Lipchitz constant for $a,\|a(x)\| \leq L_{1}\|x\|$. Then we have $x(t)^{\top} x(t) \geq x_{0}^{\top} x_{0} e^{-2 L_{1} t}, \quad \forall_{t \geq 0}$ (cf. [16, p.107, Exercise 3.17]). Thus $V(\mathrm{x}) \geq c_{1} x_{0}^{\top} x_{0}$ where $c_{1}=a_{1} \int_{0}^{t_{1}} e^{-2 L_{1} t} d t+b_{1} e^{-2 L_{1} t_{1}}>0, \forall_{t_{1}}>0$.

To see that $V(\mathrm{x}) \leq c_{2}\left\|x_{0}\right\|^{2}$, note that since (1) is MES, we have that $\mathbb{E}_{\mathrm{x}}\left[x(t)^{\top} x(t)\right] \leq c e^{-\alpha t} x_{0}^{\top} x_{0}$ for some constant $c>0$. Thus $V_{1}(\mathrm{x}) \leq a_{2} \frac{c}{\alpha} x_{0}^{\top} x_{0}$, and $V_{2}(\mathrm{x}) \leq k_{2} x_{0}^{\top} x_{0}$ where $k_{2}:=\sum_{\ell=1}^{n_{\ell}} \sum_{k=1}^{\infty} \mathbb{E}\left[b_{2} c e^{-\alpha t_{k}^{\ell}}\right]$. Note that $\mathbb{E}\left[e^{-\alpha t_{k}^{\ell}}\right]=\eta_{0} \eta^{k-1}$, where $\eta_{0}=\mathbb{E}\left[e^{-\alpha h_{0}^{\ell}}\right]$, $\eta=\mathbb{E}\left[e^{-\alpha h_{k_{1}}^{\ell}}\right]<1$, for some $1 \leq k_{1}<k$, and therefore $k_{2}<\infty$. Thus, $V(\mathrm{x}) \leq c_{2}\left\|x_{0}\right\|^{2}$ where $c_{2}=a_{2} \frac{c}{\alpha}+k_{2}$.

It follows from $[10$, p. 92 , Cor. $(32.6)])$ that $V_{1}(\mathrm{x})$ is differentiable since, as required in $[10$, p. 92, Cor. (32.6)]) $\lambda_{T}(\tau)$ is continuous. From [10, p.92, Th. (32.2)]

$$
\mathfrak{A} V_{1}(\mathrm{x})=-x_{0}^{\top} Y(\tau) x_{0}
$$

and from $[10$, p.90,91] we have that

$$
\mathbb{E}\left[V_{2}(\mathrm{x})\right]=\mathbb{E}_{\mathbf{x}}\left[\int_{0}^{\infty} \lambda_{T}(v(t)) x(t)^{\top} Z(v(t)) x(t) d t\right],
$$

from which one can conclude again from $[10$, p.92, Th. (32.2)] that

$$
\mathfrak{A} V_{2}(\mathrm{x})=-\lambda_{T}(\tau) x_{0}^{\top} Z(\tau) x_{0} .
$$

and that $V_{2}(\mathrm{x})$ is differentiable (again by [10, Cor.(32.6)]). Thus $V(\mathrm{x})$ is differentiable and $\mathfrak{A} V(\mathrm{x}) \leq-r x_{0}^{\top} x_{0}$ for $r=a_{1}$.

\section{Proof of Theorems 5 and 9}

Proof (of Theorem 5) From the explicit solution to (27), described by (44), and the commuting property, we ob- tain that

$$
\mathbb{E}\left[x(t)^{\top} x(t)\right]=x_{0}^{\top} e^{A^{\top} t} \Pi_{\ell=1}^{n_{\ell}} \mathbb{E}\left[\left(J_{\ell}^{\top}\right)^{N_{\ell}(t)} J_{\ell}^{N_{\ell}(t)}\right] e^{A t} x_{0},
$$

where $N_{\ell}(t)$ is described by (71). From [4, Th. 4], we can conclude for some symmetric matrix $C$ and for $\alpha_{\ell}$ described by (36), we have that $\mathbb{E}\left[\left(J_{\ell}^{\top}\right)^{N_{\ell}(t)} J_{\ell}^{N_{\ell}(t)}\right] \leq$ $C e^{\alpha_{\ell} t}$, if $r_{\sigma}\left(J_{\ell}^{\top} \otimes J_{\ell}^{\top}\right) \neq 0$, and therefore (76) can be bounded by $\mathbb{E}\left[x(t)^{\top} x(t)\right] \leq c e^{\left(2 \bar{\lambda}+\sum_{\ell=1}^{n_{\ell}} \alpha_{\ell}\right) t} x_{0}^{\top} x_{0}$ for some constant $c$. The result then follows from this latter observation and by noticing that if $r_{\sigma}\left(J_{\ell}^{\top} \otimes J_{\ell}^{\top}\right)=0$, the state is $x$ reset to zero after a finite number of jumps.

Proof (of Theorem 9) We rewrite the dynamic and reset maps in (1) as

$$
a(x)=A x+f_{e}(x), \quad j_{\ell}(x)=J_{\ell} x+g_{e \ell}(x),
$$

where $f_{e}(x):=a(x)-A x$, and $g_{e \ell}(x):=j_{\ell}(x)-J_{\ell} x$ are differentiable functions such that

$$
\frac{\left\|f_{e}(x)\right\|}{\|x\|} \rightarrow 0, \quad \text { and } \frac{\left\|g_{e \ell}(x)\right\|}{\|x\|} \rightarrow 0, \text { as }\|x\| \rightarrow 0
$$

(cf. [16, p.138]). Let $V(\mathbf{x}(t))=x(t)^{\top} X(v(t)) x(t)$, where $\mathbf{x}(t)$ is described by (18) and $X(\tau), \tau \in B$ satisfies (40) and $c_{1} I \leq X(\tau) \leq c_{2} I, \forall \tau \in B$ for positive constants $c_{1}$ and $c_{2}$. Then, there exists $\nu>0$ such that

$$
\mathfrak{A} V(\mathbf{x})=-r(\mathbf{x}) \leq-d_{1}\|x\|^{2}, \forall_{x:\|x\| \leq \nu}
$$

for some $d_{1}>0$, where $\mathfrak{A} V(\mathbf{x})$ is given by (23). This expression (79) can be obtained by directly replacing (77) in (23), and using (78) in a similar fashion to the proof of an analogous result for deterministic non-linear systems (cf. [16, p.139, Th. 4.7]).

Using similar arguments to [18, Th. 1,Ch. 2], we consider the stopped process $\mathbf{x}_{S}(t):=\mathbf{x}\left(t \wedge \tau_{m}\right)$, where $t \wedge \tau_{m}:=$ $\min \left(t, \tau_{m}\right) ; \tau_{m}=\inf \left\{t: \mathbf{x}(t) \notin B_{m}\right\}$ is the first exit time from the set $B_{m}:=\{\mathbf{x}: V(\mathbf{x})<m\}$; and $m \leq \frac{\nu^{2}}{c_{1}}$ is such that $\left.B_{m} \subseteq\{(x, \tau):\|x\| \leq \nu\}\right]$. It is easy to see that $\mathbf{x}\left(t \wedge \tau_{m}\right)$ is a piecewise deterministic process and from (23) and (79),

$$
\mathfrak{A} V\left(\mathbf{x}\left(t \wedge \tau_{m}\right)\right)=\left\{\begin{array}{l}
-r(\mathbf{x}) \text { if } x \in B_{m} \\
0 \text { otherwise. }
\end{array}\right.
$$

Considering (22) for the process $\mathbf{x}_{S}$ and using (80), we obtain that $\mathbb{E}_{\mathbf{x}}\left[V\left(\mathbf{x}\left(t \wedge \tau_{m}\right)\right)\right] \leq V(\mathbf{x})$, i.e., $V\left(\mathbf{x}\left(t \wedge \tau_{m}\right)\right)$ is a super-martingale, where $\mathbb{E}_{\mathrm{x}}$ denotes expectation with respect to the Markov process $\mathbf{x}$ started at initial condition $\mathrm{x}=\left(x_{0}, \tau\right)$. From this latter fact, and using the fact that $\lim _{t \rightarrow 0} \mathbb{E}_{\mathbf{x}}[V(\mathbf{x}(t))]=V(\mathrm{x})$, (cf. [10, 
p.77, Th. (27.6)]), we can apply the super-martingale theorem [18, p.26, Eq. 7.4], and conclude that

$$
\operatorname{Prob}_{\times}\left[\sup _{\infty>t \geq 0} V\left(\mathbf{x}\left(t \wedge \tau_{m}\right)\right) \geq m\right] \leq \frac{V(\mathbf{x})}{m}
$$

where Prob $_{x}$ denotes probability with respect to the Markov process $\mathbf{x}$ started at initial condition $\mathbf{x}=\left(x_{0}, \tau\right)$. Given $\epsilon, \rho$, choose $m=\frac{\min (\nu, \epsilon)^{2}}{c_{1}}$, and $\delta=\sqrt{\frac{\rho m}{c_{2}}}$. Then, for any $\left\|x_{0}\right\| \leq \delta$,

$$
\begin{aligned}
& \left.\operatorname{Prob}_{\times}\left[\sup _{\infty>t \geq 0} \| \mathbf{x}\left(t \wedge \tau_{m}\right)\right) \| \geq \epsilon\right] \leq \\
& \operatorname{Prob}_{\times}\left[\sup _{\infty>t \geq 0} V\left(\mathbf{x}\left(t \wedge \tau_{m}\right)\right) \geq m\right] \leq \frac{V(\mathbf{x})}{m} \leq \frac{c_{2}\left\|x_{0}\right\|^{2}}{m} \leq \rho
\end{aligned}
$$

i.e., the origin of (1) is stable with probability one.

\section{References}

[1] Duarte Antunes. Stochastic, Dynamic, and Periodic Networked Control Systems. PhD thesis, Instituto Superior Técnico, Lisbon, 2011. available at http://www.dct.tue.nl/ New/Antunes/phdthesis/thesis.pdf.

[2] Duarte Antunes, João P. Hespanha, and Carlos Silvestre. Stability of impulsive systems driven by renewal processes. In American Control Conference, 2009. ACC '09., pages 40324037, June 2009.

[3] Duarte Antunes, João P. Hespanha, and Carlos Silvestre. Impulsive systems triggered by superposed renewal processes. In Proc. of the 49th Conf. on Decision and Contr., Dec. 2010.

[4] Duarte Antunes, João P. Hespanha, and Carlos Silvestre. Volterra integral approach to impulsive renewal systems: Application to networked control. Automatic Control, IEEE Transactions on, 57(3):607 -619, march 2012.

[5] Richard Bellman. The stability of solutions of linear differential equations. Duke Math. J., (10):643647, 1991.

[6] Stephen Boyd and Lieven Vandenberghe. Convex Optimization. Cambridge University Press, 2004.

[7] A. Chaillet and A. Bicchi. Delay compensation in packetswitching networked controlled systems. In Decision and Control, 2008. CDC 2008. 47th IEEE Conference on, pages 3620 -3625, dec. 2008.

[8] Françoise Chatelin. Spectral approximation of linear operators. Academic Press, 1983.

[9] J. B. . Conway. A Course in Functional Analysis. SpringerVerlag, New York, 1985.

[10] M. H. A. Davis. Markov Models and Optimization. Chapman \& Hall, London, UK, 1993.

[11] M.C.F. Donkers, W.P.M.H. Heemels, D. Bernardini, A. Bemporad, and V. Shneer. Stability analysis of stochastic networked control systems. Automatica, 48(5):917 - 925, 2012.

[12] R. Goebel, R. Sanfelice, and A. Teel. Hybrid dynamical systems. Control Systems, IEEE, 29(2):28 -93, april 2009.

[13] João P. Hespanha, Payam Naghshtabrizi, and Yonggang Xu. A survey of recent results in networked control systems. Proc. of IEEE Special Issue on Technology of Networked Control Systems, 95(1):138-162, January 2007.
[14] João P. Hespanha and Andrew R. Teel. Stochastic impulsive systems driven by renewal processes. In 17th International Symposium on Mathematical Theory of Networks and Systems (MTNS06), Kyoto, Japan, July 2006.

[15] João P. Hespanha and Andrew R. Teel. Stochastic impulsive systems driven by renewal processes. In 17th International Symposium on Mathematical Theory of Networks and Systems (MTNS06), Kyoto, Japan, July 2006.

[16] Hassan Khalil. Nonlinear Systems, Third Edition. Prentice Hall, Upper Saddle River, NJ, 2002.

[17] M. A. Krasnoselskii, Je. A. Lifshits, and A. V. Sobolev. Positive linear systems : the method of positive operators. Heldermann, Verlag, Berlin, 1989.

[18] Harold J. Kushner. Stochastic stability and control. Academic Press, New York, 1967.

[19] D. A. Lawrence. Stability analysis of nonlinear sampled-data systems. In 36th IEEE Conference on Decision and Control, San Diego, CA, USA, December 1997.

[20] L.A. Montestruque and P. Antsaklis. Stability of model-based networked control systems with time-varying transmission times. Automatic Control, IEEE Transactions on, 49(9):1562-1572, Sep 2004.

[21] Daniel E. Quevedo and Dragan Nešić. Robust stability of packetized predictive control of nonlinear systems with disturbances and markovian packet losses. Automatica, 48(8):1803 - 1811, 2012.

[22] Sidney I. Resnick. Adventures in stochastic processes. Birkhauser Verlag, Basel, Switzerland, Switzerland, 1992.

[23] M. Tabbara and D. Nesic. Input output stability of networked control systems with stochastic protocols and channels. Automatic Control, IEEE Transactions on, 53(5):1160-1175, June 2008.

[24] G.C. Walsh and Hong Ye. Scheduling of networked control systems. Control Systems Magazine, IEEE, 21(1):57 -65, feb. 2001.

[25] Wei Zhang, M.S. Branicky, and S.M. Phillips. Stability of networked control systems. Control Systems Magazine, IEEE, 21(1):84 -99, feb. 2001. 